\title{
FRACTIONAL RATE OF CONVERGENCE FOR VISCOUS APPROXIMATION TO NONCONVEX CONSERVATION LAWS*
}

\author{
TAO TANG ${ }^{\dagger}$, ZHEN-HUAN TENG ${ }^{\ddagger}$, AND ZHOUPING XIN $\S$
}

\begin{abstract}
This paper considers the viscous approximations to conservation laws with nonconvex flux function. It is shown that if the entropy solutions are piecewise smooth, then the rate of $L^{1}$ convergence is a fractional number in $(0.5,1]$. This is in contrast to the corresponding result for the convex conservation laws. Numerical experiments indicate that the theoretical prediction for the convergence rate is optimal.
\end{abstract}

Key words. rate of convergence, error estimate, viscous approximation, conservation law, nonconvex flux

AMS subject classifications. 35L65, 49M25, 34A65

DOI. $10.1137 /$ S0036141001388993

1. Introduction. In this paper, we consider the initial value problem for nonconvex conservation laws

$$
\partial_{t} u+\partial_{x} f(u)=0, \quad t>0, \quad x \in \mathbf{R}
$$

which is subject to the initial condition prescribed at $t=0$,

$$
u(x, 0)=u_{0}(x)
$$

where $f \in C^{2}$. We shall investigate viscous approximations to the entropy solution of (1.1):

$$
\partial_{t} u^{\epsilon}+\partial_{x} f\left(u^{\epsilon}\right)=\epsilon \partial_{x x} u^{\epsilon}
$$

subject to the initial data

$$
u^{\epsilon}(x, 0)=u_{0}(x) .
$$

In this work, we assume that $f^{\prime \prime}(u)$ vanishes at a finite number of points. It is also assumed that the entropy solution to (1.1) and (1.2) is piecewise smooth with finitely many shock discontinuities. The existence and uniqueness of the solutions to (1.1) in the class of piecewise smooth weak solutions were studied by Ballou [1].

When the flux $f$ is convex, the solution structure for (1.1) and (1.2) has been obtained; see, e.g., Lax [10] and Dafermos [2]. If $f$ has inflection points, then the

* Received by the editors May 8, 2001; accepted for publication (in revised form) November 30, 2002; published electronically June 10, 2003.

http://www.siam.org/journals/sima/35-1/38899.html

${ }^{\dagger}$ Department of Mathematics, Hong Kong Baptist University, Kowloon Tong, Hong Kong (ttang@ math.hkbu.edu.hk). The research of this author was supported by the Hong Kong Research Grants Council.

${ }^{\ddagger}$ School of Mathematical Sciences, Peking University, Beijing 100871, People’s Republic of China (tengzh@math.pku.edu.cn). The research of this author was supported by the China State Major Key Project for Basic Research.

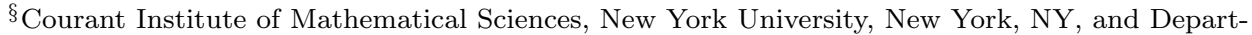
ment of Mathematics and Institute of Mathematical Sciences, The Chinese University of Hong Kong, Hong Kong (zpxin@ims.cuhk.edu.hk). The research of this author was supported by the Hong Kong Research Grants Council, NSF grants, and a DOE grant. 
situation is more complicated. In this case, some analysis for the solution structure and asymptotic behavior has been done; see, e.g., Dafermos [3], Liu [11], and Zumbrun [32]. However, we are still far from having a complete understanding of this general case, since the geometric structure of the solution, when $f$ changes convexity, is much more complicated due to the presence of contact discontinuities, and there is a large variety of asymptotic states.

The asymptotic convergence of solutions to the viscous problem (1.3) and (1.4) to the corresponding discontinuous solutions of the inviscid problem (1.1) and (1.2) has been the main driving force for the mathematical theory of shock waves from both theoretical and numerical points of view. Substantial progress has been made in the past in this regard (see [29, 20] and the references therein), pioneered by Hopf, Lax [10], Oleinik [18], and Krushkov [7], to name a few. For $B V$ entropy solutions, Kuznetsov [8] was the first to establish the half-order rate of $L^{1}$-convergence for viscosity approximation and monotone schemes. It was proved by Tang and Teng [25] that this half-order rate of convergence is optimal in the $B V$ solution class; see also Sabac [19]. However, for convex conservation laws with piecewise smooth solutions the $L^{1}$-convergence rate can be improved to first-order; see, e.g., Teng and Zhang [27] for the monotone scheme, Tang and Teng [24] for viscosity approximation, and Teng [26] for the relaxation method. The basic method in obtaining the firstorder rate of convergence is the matching asymptotic method developed by Goodman and Xin [5] and Liu and Xin [14]. One of the key ingredients in this method is the nonlinear large asymptotic stability of viscous shock profiles. For systems of viscous conservation laws, this stability theory has been extensively studied in the past decade. Important progress has been made by Goodman [4], Matsumura and Nishihara [16], Liu [12], and Szepessy and Xin [21]; see also some recent new approaches by Howard and Zumbrun [6], Liu [13], and Kreiss and Kreiss [9]. In particular, convergence with a rate to viscous shock profiles was obtained by Liu [13] by using a pointwise estimate for the approximate Green's function. Even in the case of nonconvex fluxes, the nonlinear large time asymptotic stability has been established for some special systems; see, e.g., [15] and [17]. The convergence of viscous solutions to piecewise smooth solutions for general systems was established by Goodman and Xin [5]; see also [31] for a recent improvement. For the convergence of viscous solutions in the presence of physical boundaries, we refer to [30] and the references therein. We also point out that there are some first-order pointwise convergence results for viscous approximations to convex conservation laws; see, e.g., Tadmor and Tang [22, 23], who used the energy method with some bootstrap extrapolation technique. It is proved in [24] that, for convex conservation laws whose entropy solution consists of finitely many discontinuities, the $L^{1}$-error between the viscosity solution $u^{\epsilon}$ and its inviscid limit $u$ is bounded by $\mathcal{O}(\epsilon|\ln \epsilon|)$. If neither central rarefaction waves nor spontaneous shocks occur, the error bound is improved to $\mathcal{O}(\epsilon)$; see also [28]. In this work, we will show that for nonconvex conservation laws, the $L^{1}$-error between the viscosity solution and its inviscid limit is bounded by $\mathcal{O}\left(\epsilon^{\alpha}|\ln \epsilon|\right)$, where $\frac{1}{2}<\alpha \leq 1$, even in the piecewise smooth solution class. The constant $\alpha$ is determined by the index numbers of shock curves to be defined in the next section. Based on the form of the flux function, the rate $\alpha$ can be any number between $\frac{1}{2}$ and 1 . This result suggests that for the viscous approximations the $L^{1}$-convergence rate of the nonconvex conservation laws is substantially different from that of the convex ones.

We close the introduction by outlining the rest of the paper. In the next section, we give some preliminaries, define an index number for a shock discontinuity, and list 
some properties of the index number. In section 3, we state our main convergence theorem, whose proof occupies section 4 to section 8 . Finally, in section 9 numerical experiments are performed to verify the theoretical estimates.

2. Piecewise smooth solution. Throughout this paper, we assume that the entropy solution of (1.1) and (1.2) is piecewise smooth, with finitely many shock discontinuities. More precisely, we can divide the given time interval $[0, T]$ into finite intervals $\left\{\left[t_{m-1}, t_{m}\right]\right\}_{m=1}^{M}$ such that in each interval $\left[t_{m-1}, t_{m}\right]$ the entropy solution is a finite combination of the cases plotted in Figures 1 and 2 (demonstrated in the case with three inflection points for $f(u)$ ). Thus, if we denote by $S(t)$ the set of the discontinuous curve of $u(\cdot, t)$ in the time interval $\left[t_{m-1}, t_{m}\right]$, then it consists of finitely many shocks:

$$
S(t):=\left\{(x, t) \mid x=X_{k}(t), 1 \leq k \leq K ; t_{m-1} \leq t \leq t_{m}\right\},
$$

where $X_{k}(t)<X_{k+1}(t)$ for $t \in\left(t_{m-1}, t_{m}\right)$. It is understood that $u$ is smooth with bounded limits $u\left(X_{k}(t) \pm 0, t\right)$ (denote by $\left.u_{k}^{ \pm}(t)\right)$ and $u_{x}\left(X_{k}(t) \pm 0, t\right)$. For simplicity, we will not consider the newly formed shock wave here, although this case was investigated extensively in [24]. As a consequence, we always have $u_{k}^{+}(t)-u_{k}^{-}(t) \neq 0$. For ease of notation we omit the dependence of $S(t), X_{k}(t)$, and $K$ on $m$. Each of the noncontact shocks $X_{k}(t)$, plotted in Figure 1, satisfies the Rankine-Hugoniot and the Lax conditions

$$
\begin{aligned}
& X_{k}^{\prime}(t)=\sigma\left(u_{k}^{+}(t), u_{k}^{-}(t)\right):=\frac{f\left(u_{k}^{+}(t)\right)-f\left(u_{k}^{-}(t)\right)}{u_{k}^{+}(t)-u_{k}^{-}(t)}, \\
& a\left(u_{k}^{-}(t)\right)>X_{k}^{\prime}(t)>a\left(u_{k}^{+}(t)\right), \quad \text { where } a(v):=f^{\prime}(v) .
\end{aligned}
$$

Each of the contact shocks $X_{k}(t)$, plotted in Figure 2, satisfies the Rankine-Hugoniot and the contact conditions

$$
\begin{aligned}
& X_{k}^{\prime}(t)=\sigma\left(u_{k}^{+}(t), u_{k}^{-}(t)\right), \\
& a\left(u_{k}^{-}(t)\right)>X_{k}^{\prime}(t)=a\left(u_{k}^{+}(t)\right), \quad \text { and } / \text { or } \\
& a\left(u_{k}^{-}(t)\right)=X_{k}^{\prime}(t)>a\left(u_{k}^{+}(t)\right) .
\end{aligned}
$$

We now define index numbers $\beta_{k}^{ \pm}$for a shock curve $x=X_{k}(t)$ :

1. If $X_{k}^{\prime}(t)>a\left(u_{k}^{+}\right)$, then the index number $\beta_{k}^{+}=0$.

2. If $X_{k}^{\prime}(t)=a\left(u_{k}^{+}\right)$and there exists a positive number $\beta>0$ such that

$$
\left|a\left(u_{k}^{+}\right)-a(u)\right| \sim\left|u_{k}^{+}-u\right|^{\beta} \quad \text { as } \quad u \rightarrow u_{k}^{+},
$$

then the index number $\beta_{k}^{+}=\beta$. In (2.6), the notation " $\sim$ " means equivalence. More precisely, $g(u) \sim h(u)$ as $u \rightarrow c$ means that there exists a constant $D>0$ such that $D^{-1} h \leq g \leq D h$ as $u \rightarrow c$.

3. Similarly, we can define $\beta_{k}^{-}$for the shock curve $x=X_{k}(t)$.

The following result gives a rule for calculating the index number.

THEOREM 2.1. If $f(u) \in C^{r}(\mathbf{R}), \sigma\left(u_{k}^{+}, u_{k}^{-}\right)=a\left(u_{k}^{+}\right)$, the derivative of $a(u)$ is zero at $u=u_{k}^{+}$up to $(r-1)$ th order but $a^{(r)}\left(u_{k}^{+}\right) \neq 0$, then $\beta_{k}^{+}=r$.

Proof. Applying Taylor's theorem to $a(u)$ gives

$$
a(u)-a\left(u_{k}^{+}\right)=\frac{1}{r !} a^{(r)}\left(u_{k}^{+}\right)\left(u-u_{k}^{+}\right)^{r}+o\left(\left|u-u_{k}^{+}\right|^{r}\right) .
$$



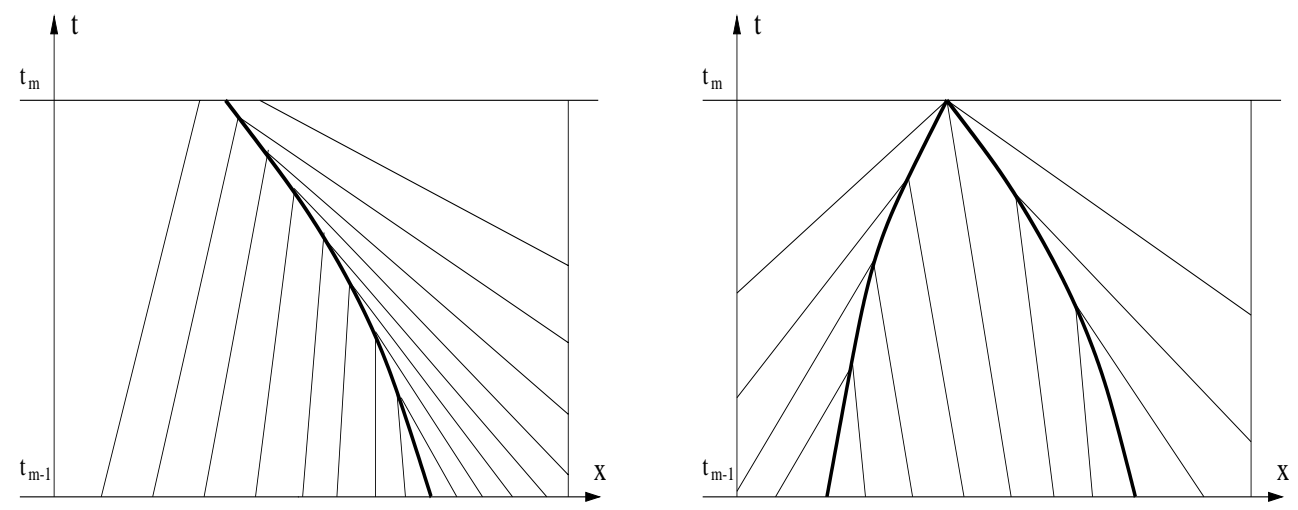

FIG. 1. Illustration of noncontact shocks: Thin lines are characteristics, and thick ones are noncontact shock curves. Here characteristics come into shocks from both sides.
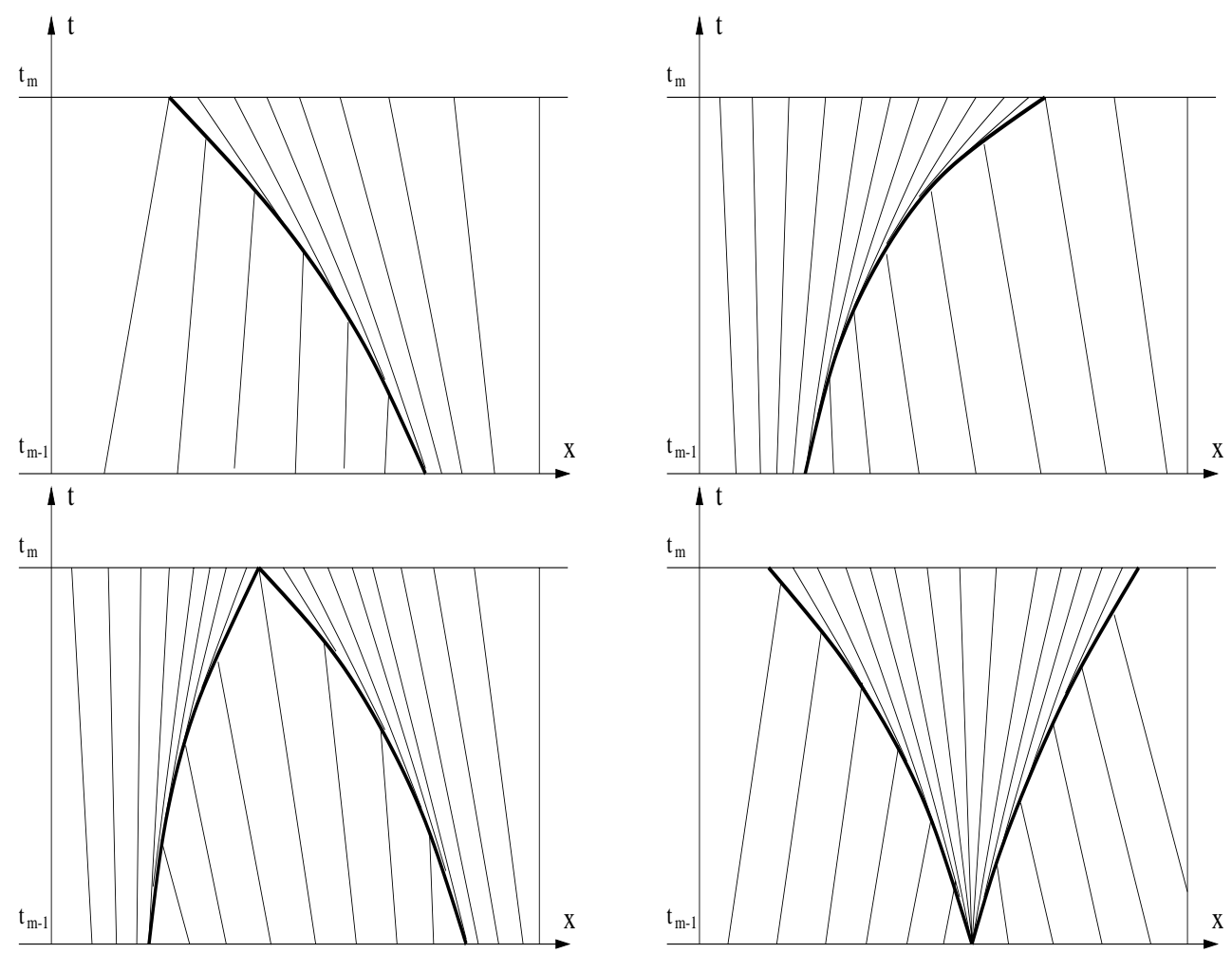

FIG. 2. Illustration of contact shocks: Thin lines are characteristics, and thick ones are contact shock curves. Here characteristics are tangent to shocks at least in one side.

This means that $\left|a(u)-a\left(u_{k}^{+}\right)\right| \sim\left|u-u_{k}^{+}\right|^{r}$ as $u \rightarrow u_{k}^{+}$. Therefore, it follows from the definition (2.6) that $\beta_{k}^{+}=r$.

The following result is an immediate consequence of the above theorem.

COROLlaRY 2.1. If $f^{\prime \prime}(u)$ has only a finite number of zero points, then the index number can take only a finite number of values. 
Example 2.1. Let $f(u)=u^{2 m+1}$. Assume the entropy solution is of the form

$$
u(x, t)= \begin{cases}u_{-} & \text {for } x<\sigma\left(u_{+}, u_{-}\right) t, \\ u_{+} & \text {for } x \geq \sigma\left(u_{+}, u_{-}\right) t\end{cases}
$$

where $u_{-}>0$ is a given number, and $u_{+}<0$ is the solution of the equation $\sigma\left(u_{+}, u_{-}\right)=a\left(u_{+}\right)$. In other words, $u_{+}$is determined by

$$
\sum_{s=0}^{2 m}\left(u_{+}\right)^{2 m-s}\left(u_{-}\right)^{s}=(2 m+1)\left(u_{+}\right)^{2 m} .
$$

It is easy to show that $a\left(u_{-}\right)>\sigma\left(u_{+}, u_{-}\right)=a\left(u_{+}\right)$, and hence $\beta^{-}=0$. Since $a^{\prime}\left(u_{+}\right)>0$, it follows from Theorem 2.1 that $\beta^{+}=1$.

Example 2.2. If $f(u)=(1-u)^{p}(1+u)^{q}$ with $p \geq 1, q \geq 1$, and $p+q>2$ and an entropy solution is given by

$$
u(x, t)= \begin{cases}-1, & x<0, \\ +1, & x \geq 0,\end{cases}
$$

then the curve $x=X(t)=0$ is a contact shock with $\sigma(1,-1)=a(1)=a(-1)$, and the index numbers are $\beta^{+}=p-1$ and $\beta^{-}=q-1$.

3. Main theorem. In this section, the main result of this paper presented; its proof will be given in the next few sections.

TheOREM 3.1. Let $f \in C^{2}$ and assume that $f^{\prime \prime}$ may change its sign at most at a finite number of points. Let $u$ be the piecewise smooth entropy solution of (1.1)(1.2) with finitely many shock discontinuities, and let $u^{\epsilon}$ be the viscosity solution of (1.3)-(1.4). Then the following error estimates hold for any $0<t \leq T$ :

$$
\left\|u^{\epsilon}(\cdot, t)-u(\cdot, t)\right\|_{L^{1}(\mathbf{R})} \leq \begin{cases}C(T) \epsilon|\ln \epsilon| & \text { for } \bar{\beta}<1 \\ C(T) \epsilon^{(1+1 / \bar{\beta}) / 2}|\ln \epsilon| & \text { for } \bar{\beta} \geq 1\end{cases}
$$

where $\bar{\beta}=\max _{0 \leq t \leq T} \beta(t), \beta=\max \left\{\beta^{+}, \beta^{-}\right\}, \beta^{ \pm}=\max _{k}\left\{\beta_{k}^{ \pm}\right\}$, and $\beta_{k}^{ \pm}$are index numbers defined by (2.6).

The above theorem will be established by using a matched asymptotic analysis, a stability lemma, and some detailed analysis for the traveling wave solution. The stability lemma to be used is valid only for the scalar conservation laws, which makes the present analysis much simpler than the system case. For the hyperbolic system, Goodman and Xin [5] constructed high-order approximations in obtaining a local first-order rate of convergence for the viscous approximations.

In the analysis of this work, we have to deal with the $L^{1}$ estimate of piecewisely continuous functions, some of which involve derivatives of some other piecewisely continuous functions; see, e.g., (7.10). In order to avoid confusion, we define the $L^{1}$-norm for a piecewisely smooth function $q$ by

$$
\|q(\cdot)\|_{\text {pis }(\mathbf{R})}=\sum_{i=1}^{I+1}\|q(\cdot)\|_{L^{1}\left(Y_{i-1}, Y_{i}\right)},
$$

where $Y_{i}$, with $Y_{0}:=-\infty$ and $Y_{I+1}:=\infty$, are all the possible discontinuous points of $q(x)$. The proof of the following stability lemma can be found in [24]. 
Lemma 3.1. Let $u^{\epsilon}$ be the viscous solution of (1.3)-(1.4). Let $v^{\epsilon} \in C(\mathbf{R} \times[0, T])$ be a piecewisely smooth function with jumps in the derivative in the set $\mathcal{A}=\{(x, t) \mid x=$ $Y_{i}(t), 1 \leq i \leq$ I\}. If $v^{\epsilon}$ satisfies

$$
\partial_{t} v^{\epsilon}+\partial_{x} f\left(v^{\epsilon}\right)=\epsilon \partial_{x x} v^{\epsilon}+g(x, t)
$$

everywhere except on the set $\mathcal{A}$, then for any $0 \leq \tau<t \leq T$

$$
\begin{aligned}
& \left\|u^{\epsilon}(\cdot, t)-v^{\epsilon}(\cdot, t)\right\|_{L^{1}(\mathbf{R})} \leq\left\|u^{\epsilon}(\cdot, \tau)-v^{\epsilon}(\cdot, \tau)\right\|_{L^{1}(\mathbf{R})} \\
& \quad+\epsilon \sum_{i=1}^{I} \int_{\tau}^{t}\left|\left[\partial_{x} v^{\epsilon}(x, t)\right]\right|_{x=Y_{i}(t)} \mid d t+\int_{\tau}^{t}\|g(\cdot, t)\|_{\text {pis }(\mathbf{R})} d t,
\end{aligned}
$$

where the jumps are defined by

$$
\left.[w(x, t)]\right|_{x=Y(t)}:=w(Y(t)+0, t)-w(Y(t)-0, t) .
$$

Remark 3.1. It will be seen in sections 7 and 8 that $g(x, t)$ in (3.2) may involve some derivatives of a discontinuous function, so we use the norm $\|\bullet\|_{\text {pis(R) }}$ to define its $L^{1}$-norm.

It follows from Theorem 3.1 that both first-order and fractional-order rates of convergence may occur for nonconvex conservation laws, which is in contrast with that for the convex conservation laws. We will demonstrate this fact with the following examples.

Example 3.1. Let $f(u)=u^{2 m+1}$. If the entropy solution $u(x, t)$ is defined by (2.7), then it follows from Example 2.1 and Theorem 3.1 that

$$
\left\|u^{\epsilon}(\cdot, t)-u(\cdot, t)\right\|_{L^{1}(\mathbf{R})} \leq C(T) \epsilon|\ln \epsilon| .
$$

Example 3.2. Let $f(u)=(1-u)^{p}(1+u)^{q}$, with $s:=\max (p, q) \geq 1$. If the entropy solution $u(x, t)$ is defined by (2.8), then it follows from Example 2.2 and Theorem 3.1 that

$$
\left\|u^{\epsilon}(\cdot, t)-u(\cdot, t)\right\|_{L^{1}(\mathbf{R})} \leq \begin{cases}C(T) \epsilon|\ln \epsilon| & \text { for } \quad 1 \leq s \leq 2 \\ C(T) \epsilon^{\frac{s}{2(s-1)}}|\ln \epsilon| & \text { for } \quad s>2\end{cases}
$$

4. Traveling wave solution of viscous equation. Our construction of an approximation solution is based on some detailed properties of viscous shock profiles for (1.3), whose nonlinear asymptotic stability was studied by Matsumura and Nishihara [15]. We will summarize some of their results in this section, which will be used in our error analysis. Some results not obtained in [15] can be derived by using the techniques developed in [24]. Let

$$
u^{\epsilon}(x, t)=V^{\epsilon}\left(x-\sigma t ; u_{+}, u_{-}\right),
$$

which is subject to the boundary conditions

$$
V^{\epsilon}\left(\xi ; u_{+}, u_{-}\right) \rightarrow u_{ \pm} \quad \text { as } \quad \xi \rightarrow \pm \infty
$$

If $V^{\epsilon}\left(x-\sigma t ; u_{+}, u_{-}\right)$satisfies (1.3), then it is called a traveling wave solution of (1.3). Applying the solution from (4.1) to (1.3) gives

$$
\epsilon V_{\xi \xi}^{\epsilon}=-\sigma V_{\xi}^{\epsilon}+f\left(V^{\epsilon}\right)_{\xi} .
$$


Integrating the above equation over $(-\infty, \xi)$ gives

$$
\epsilon V_{\xi}^{\epsilon}=-\sigma\left(V^{\epsilon}-u_{-}\right)+f\left(V^{\epsilon}\right)-f\left(u_{-}\right) .
$$

It is easy to show by rescaling $\eta=\xi / \epsilon$ that $V^{\epsilon}\left(\xi ; u_{+}, u_{-}\right)=V^{1}\left(\xi / \epsilon ; u_{+}, u_{-}\right)$. In the following we will use the notation $V\left(\eta ; u_{+}, u_{-}\right)$to denote $V^{1}\left(\eta ; u_{+}, u_{-}\right)$. We also denote by $V(\eta ; a, b)_{a}$ and $V(\eta ; a, b)_{b}$ the partial derivatives of $V$ with respect to $a$ and $b$, respectively. Note that $V^{\epsilon}\left(\xi ; u_{+}, u_{-}\right)=V\left(\xi / \epsilon ; u_{+}, u_{-}\right)$, which satisfies

$$
V^{\prime}=-\sigma\left(V-u_{-}\right)+f(V)-f\left(u_{-}\right) \text {. }
$$

It is well known that a necessary and sufficient condition for the existence of a traveling wave solution is that the constants $u_{ \pm}$and $\sigma$ satisfy the Rankine-Hugoniot condition

$$
-\sigma\left(u_{+}-u_{-}\right)+f\left(u_{+}\right)-f\left(u_{-}\right)=0
$$

and the entropy condition

$$
\Phi\left(u ; u_{+}, u_{-}\right)=:-\sigma\left(u-u_{ \pm}\right)+f(u)-f\left(u_{ \pm}\right) \begin{cases}<0 & \text { if } u_{+}<u<u_{-} \\ >0 & \text { if } u_{-}<u<u_{+}\end{cases}
$$

LEMMA 4.1. Let $u_{ \pm}$and $\sigma$ satisfy (4.5)-(4.6) and

$$
\left|\Phi\left(u ; u_{+}, u_{-}\right)\right| \sim\left|u-u_{ \pm}\right|^{1+\beta_{ \pm}} \quad \text { as } \quad u \rightarrow u_{ \pm}
$$

with $\beta_{ \pm} \geq 0$. Then there exists $V\left(\eta ; u_{+}, u_{-}\right)$, unique up to a shift, which is determined by the ordinary differential equation (4.4). Moreover, for $k=1,2$

1. if $\beta_{ \pm}=0$, then $f^{\prime}\left(u_{+}\right)<\sigma<f^{\prime}\left(u_{-}\right)$and for $\eta \rightarrow \pm \infty$

$$
\begin{aligned}
& \left|V\left(\eta ; u_{+}, u_{-}\right)-H\left(\eta ; u_{+}, u_{-}\right)\right| \sim \exp (-c|\eta|), \\
& \left|V^{(k)}\left(\eta ; u_{+}, u_{-}\right)\right| \sim \exp (-c|\eta|), \\
& \left|V\left(\eta ; u_{+}, u_{-}\right) u_{ \pm}-1\right| \sim \exp (-c|\eta|),
\end{aligned}
$$

where $H\left(\eta ; u_{+}, u_{-}\right)$is the Heaviside function satisfying $H=u_{+}$for $\eta>0$ and $H=u_{-}$for $\eta<0$;

2. if $\beta_{+}>0$, then $\sigma=f^{\prime}\left(u_{+}\right)$and for $\eta \rightarrow+\infty$

$$
\begin{aligned}
& \left|V\left(\eta ; u_{+}, u_{-}\right)-u_{+}\right| \sim|\eta|^{-1 / \beta_{+}}, \\
& \left|V^{(k)}\left(\eta ; u_{+}, u_{-}\right)\right| \sim|\eta|^{-1 / \beta_{+}-k}, \\
& \left|V\left(\eta ; u_{+}, u_{-}\right) u_{+}-1\right| \sim|\eta|^{-1 / \beta_{+}} ;
\end{aligned}
$$

3. if $\beta_{-}>0$, then $\sigma=f^{\prime}\left(u_{-}\right)$and for $\eta \rightarrow-\infty$

$$
\begin{aligned}
& \left|V\left(\eta ; u_{+}, u_{-}\right)-u_{-}\right| \sim|\eta|^{-1 / \beta_{-}}, \\
& \left|V^{(k)}\left(\eta ; u_{+}, u_{-}\right)\right| \sim|\eta|^{-1 / \beta_{-}-k}, \\
& \left|V\left(\eta ; u_{+}, u_{-}\right) u_{-}-1\right| \sim|\eta|^{-1 / \beta_{-}} .
\end{aligned}
$$

Proof. For completeness, we briefly outline the proof for this lemma. It follows from (4.4) that $V\left(\eta ; u_{+}, u_{-}\right)$can be defined implicitly by

$$
\eta=\int_{\left(u_{+}+u_{-}\right) / 2}^{V} \Phi\left(v ; u_{+}, u_{-}\right)^{-1} d v .
$$


The proof of this lemma is mainly based on the above definition and the assumption (4.7). Here we show only some of the estimates in (4.9); other estimates can be obtained similarly. The assumption (4.7) implies

$$
D^{-1}\left|v-u_{-}\right|^{1+\beta_{-}}\left|v-u_{+}\right|^{1+\beta_{+}} \leq\left|\Phi\left(v ; u_{+}, u_{-}\right)\right| \leq D\left|v-u_{-}\right|^{1+\beta_{-}}\left|v-u_{+}\right|^{1+\beta_{+}},
$$

where $D>0$ is a constant. It follows from the above inequalities and (4.11) that, for $\eta>0$,

$$
\begin{aligned}
D^{-1} 2^{-\left(1+\beta_{-}\right)} & \left|\frac{2}{u_{-}-u_{+}}\right|^{\left(1+\beta_{-}\right)}\left|\int_{\left(u_{+}+u_{-}\right) / 2}^{V}\left(v-u_{+}\right)^{-1-\beta_{+}} d v\right| \\
& \leq \eta \leq D\left|\frac{2}{u_{-}-u_{+}}\right|^{\left(1+\beta_{-}\right)}\left|\int_{\left(u_{+}+u_{-}\right) / 2}^{V}\left(v-u_{+}\right)^{-1-\beta_{+}} d v\right| .
\end{aligned}
$$

Solving the above inequalities for $V$ gives

$$
\begin{aligned}
& \left|\frac{u_{+}-u_{-}}{2}\right|\left(1+D 2^{\left(1+\beta_{-}\right)}\left|\frac{u_{+}-u_{-}}{2}\right|^{1+\beta_{+}+\beta_{-}} \beta_{+} \eta\right)^{-1 / \beta_{+}} \\
& \quad \leq\left|V-u_{+}\right| \leq\left|\frac{u_{+}-u_{-}}{2}\right|\left(1+D^{-1}\left|\frac{u_{+}-u_{-}}{2}\right|^{1+\beta_{+}+\beta_{-}} \beta_{+} \eta\right)^{-1 / \beta_{+}} .
\end{aligned}
$$

This proves the first estimate in (4.9). It is easy to show that as $\eta \rightarrow+\infty$

$$
\begin{aligned}
\left|V^{\prime}\left(\eta ; u_{+}, u_{-}\right)\right| & =\left|\Phi\left(V ; u_{+}, u_{-}\right)\right| \sim\left|V-u_{+}\right|^{1+\beta_{+}} \\
& \sim|\eta|^{-1 / \beta_{+}\left(1+\beta_{+}\right)}=|\eta|^{-1 / \beta_{+}-1} .
\end{aligned}
$$

Thus the second estimate in (4.9) follows.

COROLlary 4.1. If (4.7) holds and $\beta_{ \pm}>0$, then

$$
\left|a(u)-a\left(u_{ \pm}\right)\right| \sim\left|u-u_{ \pm}\right|^{\beta_{ \pm}} \quad \text { as } \quad u \rightarrow u_{ \pm} .
$$

COROLlaRY 4.2. Under the same assumptions as in Lemma 4.1, the following results hold:

1. If $\beta_{ \pm}=0$, then $\forall \eta \in \mathbf{R}$ and $k=1,2$

$$
\begin{aligned}
& \left|V\left(\eta ; u_{+}, u_{-}\right)-H\left(\eta ; u_{+}, u_{-}\right)\right| \leq C \exp (-c|\eta|), \\
& \left|V^{(k)}\left(\eta ; u_{+}, u_{-}\right)\right| \leq C \exp (-c|\eta|) .
\end{aligned}
$$

2. If $\beta_{+}>0$, then $\forall \eta \in \mathbf{R}^{+}$and $k=1,2$

$$
\begin{aligned}
& \left|V\left(\eta ; u_{+}, u_{-}\right)-u_{+}\right| \leq C(1+|\eta|)^{-1 / \beta_{+}} \\
& \left|V^{(k)}\left(\eta ; u_{+}, u_{-}\right)\right| \leq C(1+|\eta|)^{-1 / \beta_{+}-k} .
\end{aligned}
$$

3. If $\beta_{-}>0$, then $\forall \eta \in \mathbf{R}^{-}$and $k=1,2$

$$
\begin{aligned}
& \left|V\left(\eta ; u_{+}, u_{-}\right)-u_{-}\right| \leq C(1+|\eta|)^{-1 / \beta_{-}} \\
& \left|V^{(k)}\left(\eta ; u_{+}, u_{-}\right)\right| \leq C(1+|\eta|)^{-1 / \beta_{-}-k} .
\end{aligned}
$$


4. If $\beta_{ \pm}>0$, then $\forall \eta \in \mathbf{R}$

$$
\begin{aligned}
& \left|V\left(\eta ; u_{+}, u_{-}\right)_{u_{+}} \dot{u}_{+}+V\left(\eta ; u_{+}, u_{-}\right)_{u_{-}} \dot{u}_{-}-H\left(\eta ; \dot{u}_{+}, \dot{u}_{-}\right)\right| \\
\leq & C(1+|\eta|)^{-1 / \beta}
\end{aligned}
$$

where $\beta=\max \left\{\beta_{-}, \beta_{+}\right\}$and $\dot{w}=w^{\prime}(t)$.

Remark 4.1. It is noted that the constant $C$ in the above inequalities depends on $\left|u_{+}-u_{-}\right|^{-1}$. Since it is assumed that $u_{k}^{+}(t)-u_{k}^{-}(t) \neq 0$ on all $\left[t_{m-1}, t_{m}\right], C$ can be regarded as a constant uniform with respect to both $t \in[0, T]$ and $k=1, \ldots, K$.

5. Construction of an approximate solution. In this section, we construct an approximate solution $\widehat{u}^{\epsilon}$ to $u$ and $u^{\epsilon}$ by using the method of matching asymptotic expansions. As in [5] and [24], the main idea of constructing $\widehat{u}^{\epsilon}$ is that $\widehat{u}^{\epsilon}$ is a small perturbation of $u$ in the smooth region that posseses a viscous shock profile in places of discontinuities. We begin with the simpler case of one single shock.

5.1. An approximation to $\boldsymbol{u}$ and $\boldsymbol{u}^{\epsilon}$ with one shock. Assume that there is only one shock curve $x=X_{1}(t)$ in the entropy solution $u(x, t)$ in the time interval $\left[t_{m-1}, t_{m}\right]$. We construct a continuous approximate solution $\widehat{u}^{\epsilon}$ to $u$ and $u^{\epsilon}$ in $\left[t_{m-1}, t_{m}\right]$

$$
\widehat{u}^{\epsilon}(x, t)=m\left(\frac{x-X_{1}(t)}{\epsilon^{\gamma}}\right) I(x, t)+\left(1-m\left(\frac{x-X_{1}(t)}{\epsilon^{\gamma}}\right)\right) O(x, t),
$$

where

$$
\begin{aligned}
& I(x, t)= u(x, t)+V\left(\frac{x-X_{1}(t)}{\epsilon} ; u_{+}(t), u_{-}(t)\right) \\
&-H\left(\frac{x-X_{1}(t)}{\epsilon} ; u_{+}(t), u_{-}(t)\right), \\
& O(x, t)=u(x, t)
\end{aligned}
$$

are called first-order inner and outer solutions, respectively, $u_{ \pm}(t)=u\left(X_{1}(t) \pm 0, t\right)$, $H\left(\xi ; u_{+}, u_{-}\right)$is the Heaviside function, $0<\gamma<1$ is a constant to be determined later, $m(\xi) \in C^{\infty}(\mathbf{R})$ satisfying $0 \leq m(\xi) \leq 1$, and

$$
m(\xi)= \begin{cases}1, & |\xi| \leq 1 \\ 0, & |\xi| \geq 2\end{cases}
$$

The approximate solution can also be written in an equivalent form:

$$
\widehat{u}^{\epsilon}(x, t)=u(x, t)+m\left(\frac{x-X_{1}(t)}{\epsilon^{\gamma}}\right)(V-H)\left(\frac{x-X_{1}(t)}{\epsilon} ; u_{+}(t), u_{-}(t)\right) .
$$

The following lemma shows that $\widehat{u}^{\epsilon}$ is a good approximation to $u$ in the $L^{1}$ space.

Lemma 5.1. Assume that there is only one shock curve $x=X_{1}(t)$ for the entropy solution $u(x, t)$ in the time interval $\left[t_{m-1}, t_{m}\right]$. Then, for any $t \in\left[t_{m-1}, t_{m}\right]$,

$$
\left\|\widehat{u}^{\epsilon}(\cdot, t)-u(\cdot, t)\right\|_{L^{1}(\mathbf{R})} \leq \begin{cases}C \epsilon, & \bar{\beta}<1 \\ C \epsilon^{\gamma+(1-\gamma) / \bar{\beta}}|\ln \epsilon|, & \bar{\beta} \geq 1,\end{cases}
$$

where $\bar{\beta}=\max _{t} \beta(t)$ and $\beta=\max \left\{\beta_{1}^{+}, \beta_{1}^{-}\right\}$. 
Proof. It follows from (5.5) and (5.4) that

$$
\begin{aligned}
& \left\|\widehat{u}^{\epsilon}(\cdot, t)-u(\cdot, t)\right\|_{L^{1}(\mathbf{R})} \\
= & \int_{-2 \epsilon^{\gamma}}^{0}+\int_{0}^{2 \epsilon^{\gamma}} m\left(\frac{x}{\epsilon^{\gamma}} ; u_{+}(t), u_{-}(t)\right)\left|(V-H)\left(\frac{x}{\epsilon} ; u_{+}(t), u_{-}(t)\right)\right| d x \\
= & : I_{-}+I_{+} .
\end{aligned}
$$

Using the change of variables $\xi=x / \epsilon$ and the estimate (4.14), we have for $0<\beta_{1}^{+} \leq 1$ that

$$
\begin{aligned}
I_{+} & =\epsilon \int_{0}^{2 \epsilon^{-1+\gamma}} m\left(\epsilon^{1-\gamma} \xi\right)\left|V\left(\xi ; u_{+}, u_{-}\right)-H\left(\xi ; u_{+}, u_{-}\right)\right| d \xi \\
& =C \epsilon \int_{0}^{2 \epsilon^{-1+\gamma}}(1+|\xi|)^{-1 / \beta_{1}^{+}} d \xi \leq \begin{cases}C \epsilon, & 0<\beta_{1}^{+}<1, \\
C \epsilon|\ln \epsilon|, & \beta_{1}^{+}=1 .\end{cases}
\end{aligned}
$$

On the other hand, for $\beta_{1}^{+}>1$ using the change of variables $\xi^{\beta_{1}^{+}}=x / \epsilon$ gives

$$
\begin{aligned}
I_{+} & =\beta_{1}^{+} \epsilon \int_{0}^{2^{1 / \beta_{1}^{+}} \epsilon^{-(1-\gamma) / \beta_{1}^{+}}} m\left(\epsilon^{1-\gamma} \xi^{\beta_{1}^{+}}\right)\left|(V-H)\left(\xi^{\beta_{1}^{+}} ; u_{+}, u_{-}\right)\right| \xi^{\beta_{1}^{+}-1} d \xi \\
& \leq C \epsilon^{1-(1-\gamma)\left(\beta_{1}^{+}-1\right) / \beta_{1}^{+}} \int_{0}^{2^{1 / \beta_{1}^{+}} \epsilon^{-(1-\gamma) / \beta_{1}^{+}}}\left(1+|\xi|^{\beta_{1}^{+}}\right)^{-1 / \beta_{1}^{+}} d \xi \\
& \leq C \epsilon^{1-(1-\gamma)\left(\beta_{1}^{+}-1\right) / \beta_{1}^{+}}|\ln \epsilon| .
\end{aligned}
$$

It follows from the above results that

$$
I_{+} \leq \begin{cases}C \epsilon, & 0<\beta_{1}^{+}<1, \\ C \epsilon|\ln \epsilon|, & \beta_{1}^{+}=1, \\ C \epsilon^{1-(1-\gamma)\left(\beta_{1}^{+}-1\right) / \beta_{1}^{+}}|\ln \epsilon|, & 1<\beta_{1}^{+} .\end{cases}
$$

Similarly, we can obtain the estimates for $I_{-}$:

$$
I_{-} \leq \begin{cases}C \epsilon, & 0<\beta_{1}^{-}<1, \\ C \epsilon|\ln \epsilon|, & \beta_{1}^{-}=1, \\ C \epsilon^{1-(1-\gamma)\left(\beta_{1}^{-}-1\right) / \beta_{1}^{-}}|\ln \epsilon|, & 1<\beta_{1}^{-} .\end{cases}
$$

Combining the estimates for $I_{+}$and $I_{-}$gives the desired result (5.6).

We can also estimate the difference between $\widehat{u}^{\epsilon}$ and $u^{\epsilon}$. The result will be given below, but its proof will be deferred to section 7 .

Lemma 5.2. Assume that there is only one shock curve $x=X_{1}(t)$ for the entropy solution $u(x, t)$ in the time interval $\left[t_{m-1}, t_{m}\right]$. Then, for any $t \in\left[t_{m-1}, t_{m}\right]$,

$$
\begin{aligned}
& \left\|\widehat{u}^{\epsilon}(\cdot, t)-u^{\epsilon}(\cdot, t)\right\|_{L^{1}(\mathbf{R})} \\
\leq & \left\|\widehat{u}^{\epsilon}\left(\cdot, t_{m-1}\right)-u^{\epsilon}\left(\cdot, t_{m-1}\right)\right\|_{L^{1}(\mathbf{R})}+C \epsilon^{(1-\gamma)(\bar{\beta}+1) / \bar{\beta}}+C \epsilon^{\gamma+(1-\gamma) / \bar{\beta}} .
\end{aligned}
$$

5.2. An approximation to $u$ and $\boldsymbol{u}^{\boldsymbol{\epsilon}}$ with two shocks. Assume that in the time interval $\left[t_{m-1}, t_{m}\right]$ there exist two shock curves $x=X_{1}(t)$ and $x=X_{2}(t)$ for the entropy solution $u(x, t)$ which either collide at $t=t_{m}$, i.e., $X_{1}\left(t_{m}\right)=X_{2}\left(t_{m}\right)$, or at $t=t_{m-1}$, i.e., $X_{1}\left(t_{m-1}\right)=X_{2}\left(t_{m-1}\right)$. We construct a continuous approximate 
solution $\widehat{u}^{\epsilon}$ to $u$ and $u^{\epsilon}$ in $\left[t_{m-1}, t_{m}\right]$ by using the method of matching asymptotic expansions:

$$
\begin{aligned}
\widehat{u}^{\epsilon}(x, t)= & m\left(\frac{x-X_{1}(t)}{\epsilon^{\gamma}}\right) I_{1}(x, t)+\left(1-m\left(\frac{x-X_{1}(t)}{\epsilon^{\gamma}}\right)\right) O_{1}(x, t) \\
& +m\left(\frac{x-X_{2}(t)}{\epsilon^{\gamma}}\right) I_{2}(x, t)+\left(1-m\left(\frac{x-X_{2}(t)}{\epsilon^{\gamma}}\right)\right) O_{2}(x, t),
\end{aligned}
$$

where

$$
\begin{aligned}
& I_{i}(x, t)=u(x, t)+(V-H)\left(\frac{x-X_{i}(t)}{\epsilon} ; u_{i+}(t), u_{i-}(t)\right), \quad i=1,2, \\
& O_{i}(x, t)=u(x, t), \quad i=1,2
\end{aligned}
$$

are the first-order inner and outer solutions, respectively. Here, $u_{i \pm}(t)=u\left(X_{i}(t) \pm\right.$ $0, t), H\left(\xi ; u_{+}, u_{-}\right)$is the Heaviside function, $\gamma$ is a constant to be determined later. This approximation can be also written in an equivalent form:

$$
\begin{array}{r}
\widehat{u}^{\epsilon}(x, t)=u(x, t)+m\left(\frac{x-X_{1}(t)}{\epsilon^{\gamma}}\right)(V-H)\left(\frac{x-X_{1}(t)}{\epsilon} ; u_{1+}(t), u_{1-}(t)\right) \\
+m\left(\frac{x-X_{2}(t)}{\epsilon^{\gamma}}\right)(V-H)\left(\frac{x-X_{2}(t)}{\epsilon} ; u_{2+}(t), u_{2-}(t)\right) .
\end{array}
$$

LEMMA 5.3. Assume that in the time interval $\left[t_{m-1}, t_{m}\right]$ there exist two shock curves $x=X_{1}(t)$ and $x=X_{2}(t)$ for the entropy solution $u(x, t)$ which either collide at $t=t_{m}$ or at $t=t_{m-1}$. Then, for any $t \in\left[t_{m-1}, t_{m}\right]$,

$$
\left\|\widehat{u}^{\epsilon}(\cdot, t)-u(\cdot, t)\right\|_{L^{1}(\mathbf{R})} \leq \begin{cases}C \epsilon, & \bar{\beta}<1 \\ C \epsilon^{\gamma+(1-\gamma) / \bar{\beta}}|\ln \epsilon|, & \bar{\beta} \geq 1\end{cases}
$$

where $\bar{\beta}=\max _{t} \beta(t)$ and $\beta=\max \left\{\beta_{1}^{+}, \beta_{1}^{-}, \beta_{2}^{+}, \beta_{2}^{-}\right\}$.

LEMMA 5.4. Assume that in the time interval $\left[t_{m-1}, t_{m}\right]$ there exist two shock curves $x=X_{1}(t)$ and $x=X_{2}(t)$ for the entropy solution $u(x, t)$ which either collide at $t=t_{m}$ or at $t=t_{m-1}$. Then, for any $t \in\left[t_{m-1}, t_{m}\right]$,

$$
\begin{aligned}
& \left\|\widehat{u}^{\epsilon}(\cdot, t)-u^{\epsilon}(\cdot, t)\right\|_{L^{1}(\mathbf{R})} \\
\leq & \left\|\widehat{u}^{\epsilon}\left(\cdot, t_{m-1}\right)-u^{\epsilon}\left(\cdot, t_{m-1}\right)\right\|_{L^{1}(\mathbf{R})}+C \epsilon^{(1-\gamma)(\bar{\beta}+1) / \bar{\beta}}+C \epsilon^{\gamma+(1-\gamma) / \bar{\beta}}+C \epsilon^{2 \gamma} .
\end{aligned}
$$

The proof of Lemma 5.3 is similar to that of Lemma 5.1 and will be omitted here. We defer the proof of Lemma 5.4 to section 8 .

6. Proof of main theorem. We will prove Theorem 3.1 by considering only the case $\bar{\beta} \geq 1$, i.e., the nonconvex case; the convex result was obtained in [24]. Recall that it is assumed in each time interval $\left[t_{m-1}, t_{m}\right]$ the entropy solution $u$ is a finite combination of some noncontact shocks, contact shocks, etc. Theorem 3.1 will be established by induction on $m$. Namely, we will prove

$$
\left\|u\left(\cdot, t_{m}\right)-u^{\epsilon}\left(\cdot, t_{m}\right)\right\|_{L^{1}(\mathbf{R})} \leq \begin{cases}C(T) \epsilon|\ln \epsilon| & \text { for } \bar{\beta}<1 \\ C(T) \epsilon^{(1+1 / \bar{\beta}) / 2}|\ln \epsilon| & \text { for } \bar{\beta} \geq 1\end{cases}
$$

under the induction assumption

$$
\left\|u\left(\cdot, t_{m-1}\right)-u^{\epsilon}\left(\cdot, t_{m-1}\right)\right\|_{L^{1}(\mathbf{R})} \leq \begin{cases}C(T) \epsilon|\ln \epsilon| & \text { for } \bar{\beta}<1 \\ C(T) \epsilon^{(1+1 / \bar{\beta}) / 2}|\ln \epsilon| & \text { for } \bar{\beta} \geq 1\end{cases}
$$


The induction assumption holds for $m=1$ due to the fact $u(x, 0)=u^{\epsilon}(x, t)$. Observe that

$$
\begin{aligned}
& \left\|u\left(\cdot, t_{m}\right)-u^{\epsilon}\left(\cdot, t_{m}\right)\right\|_{L^{1}(\mathbf{R})} \\
\leq & \left\|u\left(\cdot, t_{m}\right)-\hat{v}^{\epsilon}\left(\cdot, t_{m}\right)\right\|_{L^{1}(\mathbf{R})}+\left\|\hat{v}^{\epsilon}\left(\cdot, t_{m}\right)-u^{\epsilon}\left(\cdot, t_{m}\right)\right\|_{L^{1}(\mathbf{R})} .
\end{aligned}
$$

It follows from Lemmas 5.1 and 5.3 that

$$
\left\|u\left(\cdot, t_{m}\right)-\hat{v}^{\epsilon}\left(\cdot, t_{m}\right)\right\|_{L^{1}(\mathbf{R})} \leq C \epsilon^{\gamma+(1-\gamma) / \bar{\beta}}|\ln \epsilon| .
$$

On the other hand, Lemmas 5.2 and 5.4 imply that

$$
\begin{aligned}
& \left\|\hat{v}^{\epsilon}\left(\cdot, t_{m}\right)-u^{\epsilon}\left(\cdot, t_{m}\right)\right\|_{L^{1}(\mathbf{R})} \\
\leq & \left\|\hat{v}^{\epsilon}\left(\cdot, t_{m-1}\right)-u^{\epsilon}\left(\cdot, t_{m-1}\right)\right\|_{L^{1}(\mathbf{R})}+C \epsilon^{(1-\gamma)(\bar{\beta}+1) / \bar{\beta}}+C \epsilon^{\gamma+(1-\gamma) / \bar{\beta}}+C \epsilon^{2 \gamma} .
\end{aligned}
$$

Using the induction assumption (6.2) and (6.3)-(6.5) gives

$$
\begin{aligned}
& \left\|u\left(\cdot, t_{m}\right)-u^{\epsilon}\left(\cdot, t_{m}\right)\right\|_{L^{1}(\mathbf{R})} \\
\leq & C \epsilon^{\gamma+(1-\gamma) / \bar{\beta}}|\ln \epsilon|+C \epsilon^{(1+1 / \bar{\beta}) / 2}|\ln \epsilon|+C \epsilon^{(1-\gamma)(\bar{\beta}+1) / \bar{\beta}}+C \epsilon^{\gamma+(1-\gamma) / \bar{\beta}}+C \epsilon^{2 \gamma} .
\end{aligned}
$$

Setting $\gamma=1 / 2$ in the above estimate leads to (6.1), which completes the induction proof.

7. Proof of Lemma 5.2. The main tool for establishing Lemma 5.2 is the stability lemma, Lemma 3.1. Let $v^{\epsilon}=\widehat{u}^{\epsilon}$ as defined by (5.1). Then $v^{\epsilon}$ satisfies (3.2) on its smooth region $\left\{(x, t): x \neq X_{1}(t)\right\}$, with

$$
\begin{aligned}
g(x, t)=u_{t} & +(m(V-H))_{t}+f(u+m(V-H))_{x}-\epsilon\left(u_{x x}+(m(V-H))_{x x}\right) \\
=- & a(u) u_{x}+m_{t}(V-H)-m V^{\prime} \frac{\dot{X}_{1}(t)}{\epsilon}+m(V-H)_{u_{+}} \dot{u}_{+}+m(V-H)_{u_{-}} \dot{u}_{-} \\
& +\left(a(u+m(V-H))\left(u_{x}+m_{x}(V-H)+m V^{\prime} \frac{1}{\epsilon}\right)\right. \\
& -\epsilon\left(u_{x x}+m_{x x}(V-H)+2 m_{x} V^{\prime} \frac{1}{\epsilon}+m V^{\prime \prime} \frac{1}{\epsilon^{2}}\right) .
\end{aligned}
$$

It follows from Lemma 3.1 that, for any $t \in\left[t_{m-1}, t_{m}\right]$,

$$
\begin{aligned}
& \left\|\widehat{u}^{\epsilon}(\cdot, t)-u^{\epsilon}(\cdot, t)\right\|_{L^{1}(\mathbf{R})} \\
\leq & \left\|\widehat{u}^{\epsilon}\left(\cdot, t_{m-1}\right)-u^{\epsilon}\left(\cdot, t_{m-1}\right)\right\|_{L^{1}(\mathbf{R})} \\
& +\epsilon \int_{t_{m-1}}^{t}\left|\left[\partial_{x} \widehat{u}^{\epsilon}(x, t)\right]\right|_{x=X_{1}(t)} \mid d t+\int_{t_{m-1}}^{t}\|g(\cdot, t)\|_{\mathrm{pis}(\mathbf{R})} d t \\
\leq & \left\|\widehat{u}^{\epsilon}\left(\cdot, t_{m-1}\right)-u^{\epsilon}\left(\cdot, t_{m-1}\right)\right\|_{L^{1}(\mathbf{R})}+C \epsilon+\int_{t_{m-1}}^{t}\|g(\cdot, t)\|_{\mathrm{pis}(\mathbf{R})} d t,
\end{aligned}
$$

where we have used the facts that $\left.\left[\partial_{x} \widehat{u}^{\epsilon}(x, t)\right]\right|_{x=X_{1}(t)}=\left.\left[u_{x}(x, t)\right]\right|_{x=X_{1}(t)}$ and the limits of $u_{x}(X(t) \pm 0, t)$ are uniformly bounded on $\left[t_{m-1}, t_{m}\right]$. We now claim that $g$ is 
sufficiently small such that $\widehat{u}^{\epsilon}$ satisfies (1.3) approximately. To this end, we rewrite $g$ as follows:

$$
\begin{aligned}
g(x, t)=m & \left(\left(a(u+m(V-H))-\dot{X}_{1}(t)\right) V^{\prime}-V^{\prime \prime}\right) \epsilon^{-1} \\
& +(a(u+m(V-H))-a(u)) u_{x} \\
& +\left(\left(a(u+m(V-H))-\dot{X}_{1}(t)\right) m^{\prime}-m^{\prime \prime} \epsilon^{1-\gamma}\right) \epsilon^{-\gamma}(V-H) \\
& -2 m^{\prime} \epsilon^{-\gamma} V^{\prime}-\epsilon u_{x x}+m(V-H)_{u_{+}} \dot{u}_{+}+m(V-H)_{u_{-}} \dot{u}_{-} \\
=(a & \left.(u+m(V-H))-\dot{X}_{1}(t)\right)\left(m V^{\prime} \epsilon^{-1}+u_{x}+m^{\prime}(V-H) \epsilon^{-\gamma}\right) \\
& -m V^{\prime \prime} \epsilon^{-1}+\left(\dot{X}_{1}(t)-a(u)\right) u_{x}-m^{\prime \prime}(V-H) \epsilon^{1-2 \gamma}-2 m^{\prime} \epsilon^{-\gamma} V^{\prime}-\epsilon u_{x x} \\
& +m\left(V_{u_{+}} \dot{u}_{+}+V_{u_{-}} \dot{u}_{-}-H\left(x-X_{1}(t) ; \dot{u}_{+}(t), \dot{u}_{-}(t)\right)\right) .
\end{aligned}
$$

Using the traveling wave equation $V^{\prime \prime}=\left(a(V)-\dot{X}_{1}(t)\right) V^{\prime}$ gives

$$
\begin{aligned}
g(x, t)=( & (u+m(V-H))-a(V)) m V^{\prime} \epsilon^{-1} \\
& +\left(a(u+m(V-H))-\dot{X}_{1}(t)\right)\left(u_{x}+m^{\prime}(V-H) \epsilon^{-\gamma}\right) \\
& +\left(\dot{X}_{1}(t)-a(u)\right) u_{x}-m^{\prime \prime}(V-H) \epsilon^{1-2 \gamma}-2 m^{\prime} \epsilon^{-\gamma} V^{\prime}-\epsilon u_{x x} \\
& +m\left(V_{u_{+}} \dot{u}_{+}+V_{u_{-}} \dot{u}_{-}-H\left(x-X_{1}(t) ; \dot{u}_{+}(t), \dot{u}_{-}(t)\right)\right) .
\end{aligned}
$$

Without loss of generality, we will consider only contact shock curves, i.e., $\beta_{1}^{ \pm} \geq 1$. The assumption $\beta_{1}^{ \pm} \geq 1$ implies that $\dot{X}_{1}(t)=a\left(u_{ \pm}\right)$, which leads to

$$
\begin{aligned}
g(x, t)=( & a(u+m(V-H))-a(V)) m V^{\prime} \epsilon^{-1} \\
& +\left(a(u+m(V-H))-a\left(u_{ \pm}\right)\right)\left(u_{x}+m^{\prime}(V-H) \epsilon^{-\gamma}\right) \\
& +\left(a\left(u_{ \pm}\right)-a(u)\right) u_{x}-m^{\prime \prime}(V-H) \epsilon^{1-2 \gamma}-2 m^{\prime} \epsilon^{-\gamma} V^{\prime}-\epsilon u_{x x} \\
& +m\left(V_{u_{+}} \dot{u}_{+}+V_{u_{-}} \dot{u}_{-}-H\left(x-X_{1}(t) ; \dot{u}_{+}(t), \dot{u}_{-}(t)\right)\right) .
\end{aligned}
$$

We now split $\|g(\cdot, t)\|_{\text {pis(R) }}$ into the following three parts:

$$
\begin{aligned}
\|g(\cdot, t)\|_{\operatorname{pis}(\mathbf{R})} & =\int_{0<\left|x-X_{1}(t)\right| \leq \epsilon^{\gamma}}+\int_{\epsilon^{\gamma} \leq\left|x-X_{1}(t)\right| \leq 2 \epsilon^{\gamma}}+\int_{\left|x-X_{1}(t)\right| \geq 2 \epsilon^{\gamma}}|g(x, t)| d t \\
& =I+I I+I I I .
\end{aligned}
$$

7.1. Piecewise constant solution. In order to estimate $I, I I$, and $I I I$ above we first consider a simple but important case: the piecewise constant solution, i.e.,

$$
u(x, t)= \begin{cases}u_{+}, & x>X_{1}(t) \\ u_{-}, & x \leq X_{1}(t)\end{cases}
$$

where $u_{+}$and $u_{-}$are constants and $\dot{X}_{1}(t)=\left(f\left(u_{-}\right)-f\left(u_{+}\right)\right) /\left(u_{-}-u_{+}\right)$. It is easy to show that $g(x, t)=0$ for $0<\left|x-X_{1}(t)\right| \leq \epsilon^{\gamma}$ and $\left|x-X_{1}(t)\right|>2 \epsilon^{\gamma}$. Therefore $I=I I I=0$, and what we need to estimate is the term $I I$. Let

$$
I I=I I_{+}+I I_{-}
$$

where 


$$
\begin{gathered}
I I_{ \pm}=: \pm \int_{X_{1}(t) \pm \epsilon^{\gamma}}^{X_{1}(t) \pm 2 \epsilon^{\gamma} \mid}\left(a\left(u_{ \pm}+m\left(V-u_{ \pm}\right)\right)-a(V)\right) m V^{\prime} \epsilon^{-1} \\
+\left(a\left(u_{ \pm}+m\left(V-u_{ \pm}\right)\right)-a\left(u_{ \pm}\right)\right) m^{\prime}\left(V-u_{ \pm}\right) \epsilon^{-\gamma} \\
-m^{\prime \prime}\left(V-u_{ \pm}\right) \epsilon^{1-2 \gamma}-2 m^{\prime} \epsilon^{-\gamma} V^{\prime} \mid d x \\
= \pm \int_{X_{1}(t) \pm \epsilon^{\gamma}}^{X_{1}(t) \pm 2 \epsilon^{\gamma}} \mid\left(a\left(u_{ \pm}+m\left(V-u_{ \pm}\right)\right)-a\left(u_{ \pm}\right)\right)\left(m V^{\prime} \epsilon^{-1}+m^{\prime}\left(V-u_{ \pm}\right) \epsilon^{-\gamma}\right) \\
-\left(a(V)-a\left(u_{ \pm}\right)\right) m V^{\prime} \epsilon^{-1}-m^{\prime \prime}\left(V-u_{ \pm}\right) \epsilon^{1-2 \gamma}-2 m^{\prime} \epsilon^{-\gamma} V^{\prime} \mid d x
\end{gathered}
$$

Using the change of variables $\xi=\left(x-X_{1}(t)\right) / \epsilon^{\gamma}$ gives

$$
\begin{aligned}
I I_{ \pm}= \pm \int_{ \pm 1}^{ \pm 2} \mid & \left(a\left(u_{ \pm}+m\left(V-u_{ \pm}\right)\right)-a\left(u_{ \pm}\right)\right)\left(m V^{\prime} \epsilon^{-1+\gamma}+m^{\prime}\left(V-u_{ \pm}\right)\right) \\
& -\left(a(V)-a\left(u_{ \pm}\right)\right) m V^{\prime} \epsilon^{-1+\gamma}-m^{\prime \prime}\left(V-u_{ \pm}\right) \epsilon^{1-\gamma}-2 m^{\prime} V^{\prime} \mid d \xi
\end{aligned}
$$

where $m=m(\xi)$ and $V=V\left(\xi / \epsilon^{1-\gamma}\right)$. The following estimates can be obtained from Lemma 4.1 and Corollary 4.1:

$$
\begin{aligned}
& \left|V-u_{ \pm}\right| \leq C \epsilon^{(1-\gamma) / \beta_{1}^{ \pm}}, \quad\left|V^{\prime}\right| \leq C \epsilon^{(1-\gamma)\left(1+1 / \beta_{1}^{ \pm}\right)} \\
& \left|a\left(u_{ \pm}+m\left(V-u_{ \pm}\right)\right)-a\left(u_{ \pm}\right)\right| \leq C\left|V-u_{ \pm}\right|^{\beta_{1}^{ \pm}} \leq C \epsilon^{1-\gamma} \\
& \left|a(V)-a\left(u_{ \pm}\right)\right| \leq C\left|V-u_{ \pm}\right|^{\beta_{1}^{ \pm}} \leq C \epsilon^{1-\gamma}
\end{aligned}
$$

provided $\epsilon$ is sufficiently small. It follows from (7.8) and the above estimates that

$$
I I_{ \pm} \leq C \epsilon^{(1-\gamma)\left(1+1 / \beta_{1}^{ \pm}\right)} .
$$

The above results, together with the facts $I=I I I=0$, give the desired upper bound for $\|g(\cdot, t)\|_{\text {pis }(\mathbf{R})}$. Therefore, Lemma 5.2 is established in the case of the piecewise constant solution.

7.2. Piecewise smooth solution. We now consider a more general case, i.e., $u$ is piecewise smooth. It follows from (7.3) that $g(x, t)=-\epsilon u_{x x}$ for $\left|x-X_{1}(t)\right|>2 \epsilon^{\gamma}$, and

$$
\begin{aligned}
g(x, t)= & (a(u+(V-H))-a(V)) V^{\prime} \epsilon^{-1} \\
& +(a(u+(V-H))-a(u)) u_{x}-\epsilon u_{x x} \\
& +V_{u_{+}} \dot{u}_{+}+V_{u_{-}} \dot{u}_{-}-H\left(x-X_{1}(t) ; \dot{u}_{+}, \dot{u}_{-}\right)
\end{aligned}
$$

for $0<\left|x-X_{1}(t)\right| \leq \epsilon^{\gamma}$. It is easy to see from (7.4) that

$$
I I I=\epsilon \int_{\left|x-X_{1}(t)\right| \geq 2 \epsilon^{\gamma}}\left|u_{x x}\right| d x \leq C \epsilon,
$$

where $u_{x x}(\cdot, t)$ is assumed piecewisely in $L^{1}$. It follows from (7.10) that

$$
I \leq I_{1}+I_{2}+I_{3}+I_{4}
$$


where

$$
\begin{aligned}
& I_{1}=\int_{0<\left|x-X_{1}(t)\right| \leq \epsilon^{\gamma}}-|a(u+(V-H))-a(V)| V^{\prime} \epsilon^{-1} d x, \\
& I_{2}=\int_{0<\left|x-X_{1}(t)\right| \leq \epsilon^{\gamma}}\left|(a(u+(V-H))-a(u)) u_{x}\right| d x, \\
& I_{3}=\int_{0<\left|x-X_{1}(t)\right| \leq \epsilon^{\gamma}} \epsilon\left|u_{x x}\right| d x, \\
& I_{4}=\int_{0<\left|x-X_{1}(t)\right| \leq \epsilon^{\gamma}}\left|V_{u_{+}} \dot{u}_{+}+V_{u_{-}} \dot{u}_{-}-H\left(x-X_{1}(t) ; \dot{u}_{+}, \dot{u}_{-}\right)\right| d x .
\end{aligned}
$$

We now estimate $I_{i}, 1 \leq i \leq 4$. Since $u$ is piecewise smooth and $u-H \rightarrow 0$ as $x \rightarrow X_{1}(t) \pm 0$, we have

$$
\begin{aligned}
|a(u+(V-H))-a(V)| & =\left|a^{\prime}(V+\theta(u-H))(u-H)\right| \\
& \leq C\left|x-X_{1}(t)\right| .
\end{aligned}
$$

Therefore, we can find a positive function $A(\xi)$ such that $\forall x \in(-\infty, \infty)$

- $|a(u+(V-H))-a(V)| \leq A\left(x-X_{1}(t)\right)$

- $A(\xi) \leq C|\xi|$

- $\left|A^{\prime}(\xi)\right| \leq M$,

where $M$ is a constant. It follows from the above auxiliary function $A$ and the estimates (4.9) and (4.10) that

$$
\begin{aligned}
I_{1} \leq & \int_{\left|x-X_{1}(t)\right| \leq \epsilon^{\gamma}}-A\left(x-X_{1}(t)\right) V^{\prime}\left(\left(x-X_{1}(t)\right) / \epsilon\right) \epsilon^{-1} d x \\
& =\int_{-\epsilon^{\gamma-1}}^{\epsilon^{\gamma-1}}-A(\epsilon \xi) V^{\prime}(\xi) d \xi \\
& \left.=\int_{-\epsilon^{\gamma-1}}^{\epsilon^{\gamma-1}}-A(\epsilon \xi)(V(\xi)-H(\xi))^{\prime} d \xi \quad \text { (using the fact } A(0)=0\right) \\
\leq & A\left(\epsilon^{\gamma}\right)\left|V\left(\epsilon^{\gamma-1}\right)-H\left(\epsilon^{\gamma-1}\right)\right|+A\left(-\epsilon^{\gamma}\right)\left|V\left(-\epsilon^{\gamma-1}\right)-H\left(-\epsilon^{\gamma-1}\right)\right| \\
& \quad+M \epsilon \int_{-\epsilon^{\gamma-1}}^{\epsilon^{\gamma-1}}|V(\xi)-H(\xi)| d \xi \quad \text { (using integration by parts) } \\
& \leq C \epsilon^{\gamma+(1-\gamma) / \beta}+C \epsilon^{1-(1-\gamma)(1-1 / \beta)} \leq \epsilon^{\gamma+(1-\gamma) / \beta} .
\end{aligned}
$$

Observe that $|a(u+(V-H))-a(u)| \leq C|V-H|$, which, if applied to $I_{2}$, gives

$$
\begin{aligned}
I_{2} & \leq C \int_{\left|x-X_{1}(t)\right| \leq \epsilon^{\gamma}}|V-H| d x \\
& \leq C \epsilon \int_{-\epsilon^{\gamma-1}}^{\epsilon^{\gamma-1}}|V(\xi)-H(\xi)| d \xi \\
& \leq C \epsilon \int_{-\epsilon^{\gamma-1}}^{\epsilon^{\gamma-1}}(1+|\xi|)^{-1 / \beta} d \xi \\
& \leq C \epsilon^{1-(1-\gamma)(1-1 / \beta)}=C \epsilon^{\gamma+(1-\gamma) / \beta} .
\end{aligned}
$$


Moreover, by the definition of $I_{3}$ we can easily obtain $I_{3} \leq C \epsilon^{1+\gamma}$. Using the change of variables $\xi=\left(x-X_{1}(t)\right) / \epsilon$ and the estimate (4.16) gives

$$
I_{4} \leq C \epsilon \int_{-\epsilon^{\gamma-1}}^{\epsilon^{\gamma-1}}(1+|\xi|)^{-1 / \beta} d \xi \leq C \epsilon^{1-(1-\gamma)(1-1 / \beta)}=C \epsilon^{\gamma+(1-\gamma) / \beta} .
$$

Combining the above estimates yields

$$
I \leq C\left(\epsilon^{\gamma+(1-\gamma) / \beta}+\epsilon^{\gamma(1+\beta)}+\epsilon|\ln \epsilon|+\epsilon^{(1+\gamma)}\right) .
$$

It remains to estimate $I I$. It follows from (7.3) that

$$
I I \leq I I_{+}+I I_{-}+\sum_{i=1}^{5} I I_{+}^{(i)}+\sum_{i=1}^{5} I I_{-}^{(i)}
$$

where $I I_{ \pm}$is defined by (7.7) and

$$
\begin{aligned}
& I_{ \pm}^{(1)}= \pm \int_{X_{1}(t) \pm \epsilon^{\gamma}}^{X_{1}(t) \pm 2 \epsilon^{\gamma}}\left|\left(a(u+m(V-H))-a\left(u_{ \pm}+m(V-H)\right)\right) m V^{\prime}\right| \epsilon^{-1} d x \\
& I_{ \pm}^{(2)}= \pm \int_{X_{1}(t) \pm \epsilon^{\gamma}}^{X_{1}(t) \pm 2 \epsilon^{\gamma}}\left|(a(u+m(V-H))-a(u)) u_{x}\right| d x \\
& I I_{ \pm}^{(3)}= \pm \int_{X_{1}(t) \pm \epsilon^{\gamma}}^{X_{1}(t) \pm 2 \epsilon^{\gamma}}\left|\left(a(u+m(V-H))-a\left(u_{ \pm}+m(V-H)\right)\right) m^{\prime}(V-H)\right| \epsilon^{-\gamma} d x \\
& I I_{ \pm}^{(4)}= \pm \int_{X_{1}(t) \pm \epsilon^{\gamma}}^{X_{1}(t) \pm 2 \epsilon^{\gamma}} \epsilon\left|u_{x x}\right| d x \\
& I I_{ \pm}^{(5)}= \pm \int_{X_{1}(t) \pm \epsilon^{\gamma}}^{X_{1}(t) \pm 2 \epsilon^{\gamma}} m\left|V_{u_{+}} \dot{u}_{+}+V_{u_{-}} \dot{u}_{-}-H\left(x-X_{1}(t) ; \dot{u}_{+}, \dot{u}_{-}\right)\right| d x .
\end{aligned}
$$

It follows from (7.9) that

$$
I I_{ \pm} \leq C \epsilon^{(1-\gamma)\left(1+1 / \beta_{1}^{ \pm}\right)} .
$$

The estimate for $I I_{ \pm}^{(1)}$ is similar to that for $I_{1}$, with the same error bound as (7.13), namely,

$$
I I_{ \pm}^{(1)} \leq C \epsilon^{\gamma+(1-\gamma) / \beta}
$$

The estimate for $I I_{ \pm}^{(2)}$ is similar to that for $I_{2}$, with the same error bound as (7.14), namely,

$$
I I_{ \pm}^{(2)} \leq C\left(\epsilon^{\gamma(1+\beta)}+\epsilon|\ln \epsilon|\right)
$$

The estimate for $I I_{ \pm}^{(5)}$ is similar to that for $I_{4}$, with the same error bound as (7.15), namely,

$$
I I_{ \pm}^{(5)} \leq C \epsilon^{1-(1-\gamma)(1-1 / \beta)}=C \epsilon^{\gamma+(1-\gamma) / \beta} .
$$


Using the facts that $a(u+m(V-H))-a\left(u_{ \pm}+m(V-H)\right)=a^{\prime}(\bullet)\left(u-u_{ \pm}\right)$, and $\left|u-u_{ \pm}\right| \leq C\left|x-X_{1}(t)\right|$ for $\pm\left(x-X_{1}(t)\right)>0$, we obtain

$$
I I_{ \pm}^{(3)} \leq \pm C \epsilon^{\gamma} \int_{X_{1}(t) \pm \epsilon^{\gamma}}^{X_{1}(t) \pm 2 \epsilon^{\gamma}}|(V-H)| \epsilon^{-\gamma} d x
$$

Applying the change of variables $\left(x-X_{1}(t)\right) / \epsilon=\xi$ to the above integration gives

$$
I I_{ \pm}^{(3)} \leq \pm C \epsilon \int_{ \pm \epsilon^{\gamma-1}}^{ \pm 2 \epsilon^{\gamma-1}}|V(\xi)-H(\xi)| d \xi
$$

It then follows from (4.14) and (4.15) that

$$
\begin{aligned}
I I_{ \pm}^{(3)} & \leq \pm C \epsilon \int_{ \pm \epsilon^{\gamma-1}}^{ \pm 2 \epsilon^{\gamma-1}}(1+|\xi|)^{-1 / \beta_{1}^{ \pm}} d \xi \leq C \epsilon^{1-(1-\gamma)\left(1-1 / \beta_{1}^{ \pm}\right)} \\
& =C \epsilon^{\gamma+(1-\gamma) / \beta_{1}^{ \pm}}
\end{aligned}
$$

Moreover, using the definition of $I I_{ \pm}^{(4)}$ gives

$$
I I_{ \pm}^{(4)} \leq \pm C \int_{X_{1}(t) \pm \epsilon^{\gamma}}^{X_{1}(t) \pm 2 \epsilon^{\gamma}} \epsilon\left|u_{x x}\right| d x \leq C \epsilon^{1+\gamma}
$$

Combining the estimates (7.17)-(7.22) leads to

$$
\begin{aligned}
I I & \leq C\left(\epsilon^{(1-\gamma)(1+1 / \beta)}+\epsilon^{\gamma+(1-\gamma) / \beta)}+\epsilon^{\gamma(1+\beta)}+\epsilon|\ln \epsilon|+\epsilon^{1+\gamma}\right) \\
& \leq C\left(\epsilon^{(1-\gamma)(1+1 / \beta)}+\epsilon^{\gamma+(1-\gamma) / \beta)}\right) .
\end{aligned}
$$

Adding the estimates for $I, I I$, and $I I I$ gives

$$
\begin{aligned}
\|g(\cdot, t)\|_{\operatorname{pis}(\mathbf{R})} & \leq C\left(\epsilon^{\gamma+(1-\gamma) / \beta}+\epsilon^{(1-\gamma)(1+1 / \beta)}\right) \\
& \leq C\left(\epsilon^{\gamma+(1-\gamma) / \bar{\beta}}+\epsilon^{(1-\gamma)(1+1 / \bar{\beta})}\right) .
\end{aligned}
$$

This completes the proof for Lemma 5.2.

8. Proof of Lemma 5.4. The main difference between Lemmas 5.2 and 5.4 is that Lemma 5.2 deals with only one shock, while the latter deals with two interacting shocks. The main tool for the proof of Lemma 5.4 is still the stability lemma, Lemma 3.1. Let $v^{\epsilon}=\widehat{u}^{\epsilon}$, which is defined by (5.15). Then $v^{\epsilon}$ satisfies (3.2) on its smooth region $\left\{(x, t): x \neq X_{i}(t), i=1,2\right\}$, with

$$
\begin{aligned}
g(x, t)= & \left(a\left(u+m_{1}\left(V_{1}-H_{1}\right)+m_{2}\left(V_{2}-H_{2}\right)\right)-a(u)\right) u_{x} \\
& +m_{1}^{\prime}\left(a\left(u+m_{1}\left(V_{1}-H_{1}\right)+m_{2}\left(V_{2}-H_{2}\right)\right)-a\left(H_{1}\right)\right)\left(V_{1}-H_{1}\right) \epsilon^{-\gamma} \\
& +m_{2}^{\prime}\left(a\left(u+m_{1}\left(V_{1}-H_{1}\right)+m_{2}\left(V_{2}-H_{2}\right)\right)-a\left(H_{2}\right)\right)\left(V_{2}-H_{2}\right) \epsilon^{-\gamma} \\
& +m_{1}\left(a\left(u+m_{1}\left(V_{1}-H_{1}\right)+m_{2}\left(V_{2}-H_{2}\right)\right)-a\left(V_{1}\right)\right) V_{1}^{\prime} \epsilon^{-1} \\
& +m_{2}\left(a\left(u+m_{1}\left(V_{1}-H_{1}\right)+m_{2}\left(V_{2}-H_{2}\right)\right)-a\left(V_{2}\right)\right) V_{2}^{\prime} \epsilon^{-1} \\
& -\epsilon u_{x x}-\left(m_{1}^{\prime \prime}\left(V_{1}-H_{1}\right)+m_{2}^{\prime \prime}\left(V_{2}-H_{2}\right)\right) \epsilon^{1-2 \gamma}-2\left(m_{1}^{\prime} V_{1}^{\prime}+m_{2}^{\prime} V_{2}^{\prime}\right) \epsilon^{-\gamma} \\
& +m_{1}\left(V_{1 u_{+}} \dot{u}_{1+}+V_{1 u_{-}} \dot{u}_{1-}-\dot{H}_{1}\right)+m_{2}\left(V_{2 u_{+}} \dot{u}_{2+}+V_{2 u_{-}} \dot{u}_{2-}-\dot{H}_{2}\right) \\
:= & g_{1}+g_{2}+g_{3}+g_{4}+g_{5}+g_{6}+g_{7},
\end{aligned}
$$


where for $i=1,2$

$$
\begin{aligned}
& m_{i}=m\left(\left(x-X_{i}(t)\right) / \epsilon^{\gamma}\right), \quad u_{i \pm}(t)=\lim _{x \rightarrow X_{i} \pm 0} u(x, t), \\
& \dot{u}_{i \pm}(t)=\frac{d}{d t} u_{i \pm}(t), \quad V_{i}=V\left(\left(x-X_{i}(t)\right) / \epsilon ; u_{i+}, u_{i-}\right), \\
& H_{i}=H\left(\left(x-X_{i}(t)\right) / \epsilon ; u_{i+}, u_{i-}\right), \quad \dot{H}_{i}=H\left(\left(x-X_{i}(t)\right) / \epsilon ; \dot{u}_{i+}, \dot{u}_{i-}\right) .
\end{aligned}
$$

Similarly, it follows from Lemma 3.1 that, for any $t \in\left[t_{m-1}, t_{m}\right]$,

$$
\begin{aligned}
& \left\|\widehat{u}^{\epsilon}(\cdot, t)-u^{\epsilon}(\cdot, t)\right\|_{L^{1}(\mathbf{R})} \\
\leq & \left\|\widehat{u}^{\epsilon}\left(\cdot, t_{m-1}\right)-u^{\epsilon}\left(\cdot, t_{m-1}\right)\right\|_{L^{1}(\mathbf{R})} \\
& +\epsilon \sum_{i=1}^{2} \int_{t_{m-1}}^{t}\left|\left[\partial_{x} \widehat{u}^{\epsilon}(x, t)\right]\right|_{x=X_{i}(t)} \mid d t+\int_{t_{m-1}}^{t}\|g(\cdot, t)\|_{\text {pis }(\mathbf{R})} d t \\
\leq & \left\|\widehat{u}^{\epsilon}\left(\cdot, t_{m-1}\right)-u^{\epsilon}\left(\cdot, t_{m-1}\right)\right\|_{L^{1}(\mathbf{R})}+C \epsilon+\int_{t_{m-1}}^{t}\|g(\cdot, t)\|_{\operatorname{pis}(\mathbf{R})} d t .
\end{aligned}
$$

In order to estimate the last term above we need to estimate $\int_{t_{m-1}}^{t}\left\|g_{i}(\cdot, t)\right\|_{\text {pis(R) }} d t$, $1 \leq i \leq 7$. It is noticed that the estimates for $\left\|g_{6}\right\|_{\mathrm{pis}(\mathbf{R})}$ and $\left\|g_{7}\right\|_{\text {pis }(\mathbf{R})}$ are similar to those in one shock case, so they can be bounded above by the right-hand side of (7.24). Therefore, we just need to estimate $\left\|g_{i}\right\|_{\text {pis(R) }}$ for $1 \leq i \leq 5$.

In what follows we assume that the two shock curves start at a same point, i.e.,

$$
X_{1}\left(t_{m-1}+0\right)=X_{2}\left(t_{m-1}+0\right),
$$

but do not become tangent to each other at this point:

$$
\dot{X}_{1}\left(t_{m-1}+0\right)<\dot{X}_{2}\left(t_{m-1}+0\right) .
$$

This implies that there is a constant $c>0$ such that

$$
\delta(t):=X_{2}(t)-X_{1}(t) \geq c\left(t-t_{m-1}\right) \quad \text { for } \quad t \in\left[t_{m-1}, t_{m}\right] .
$$

Note that the support of $m\left(\left(\cdot-X_{i}(t)\right) / \epsilon^{\gamma}\right)$ is $\left[X_{i}(t)-2 \epsilon^{\gamma}, X_{i}(t)+2 \epsilon^{\gamma}\right]$. Hence, if $X_{2}(t)-X_{1}(t) \geq 4 \epsilon^{\gamma}$, then for each $x \in \mathbf{R}$ only one of $m_{1}$ and $m_{2}$ appears in $g(x, t)$. Therefore, when $X_{2}(t)-X_{1}(t) \geq 4 \epsilon^{\gamma}$, the estimates for $\left\|g_{i}\right\|_{L^{1}}, 1 \leq i \leq 5$, are similar to those for the one shock case. Let $\tau \in\left(t_{m-1}, t_{m}\right)$ such that

$$
X_{2}(\tau)-X_{1}(\tau)=4 \epsilon^{\gamma} \quad \text { and } \quad X_{2}(t)-X_{1}(t)>4 \epsilon^{\gamma} \quad \text { for } \quad t \in\left(\tau, t_{m}\right]
$$

The above analysis implies that we only need to estimate $\left\|g_{i}\right\|_{L^{1}}, 1 \leq i \leq 5$, for $t \in\left[t_{m-1}, \tau\right]$.

We first estimate $\left\|g_{1}\right\|_{L^{1}}$ for $t \in\left[t_{m-1}, \tau\right]$. Observe that

$$
\begin{aligned}
\left\|g_{1}(\cdot, t)\right\|_{\operatorname{pis}(\mathbf{R})=} & \int_{\left\{x \leq X_{1}(t)-2 \epsilon^{\gamma}\right\} \cup\left\{x \geq X_{2}(t)+2 \epsilon^{\gamma}\right\}}\left|g_{1}\right| d x \\
& +\int_{X_{1}(t)-2 \epsilon^{\gamma}<x<X_{2}(t)+2 \epsilon^{\gamma}}\left|g_{1}\right| d x \\
:= & G_{11}+G_{12} .
\end{aligned}
$$


Since only one of $m_{1}$ and $m_{2}$ appears in $G_{11}$, the estimate of $G_{11}$ is similar to that of $I I_{ \pm}^{(2)}$ in the one shock case, and it follows from (7.19) that

$$
G_{11} \leq C\left(\epsilon^{\gamma(1+\beta)}+\epsilon|\ln \epsilon|\right),
$$

where $\beta=\max \left\{\beta_{1}, \beta_{2}\right\}$. The integrand of $G_{12}$ is bounded, and therefore

$$
G_{12} \leq C\left(X_{2}(t)-X_{1}(t)+\epsilon^{\gamma}\right) .
$$

It follows from (8.5), (8.4), and (8.2) that $\tau-t_{m-1} \leq C \epsilon^{\gamma}$ and $X_{2}(t)-X_{1}(t) \leq$ $C\left(t-t_{m-1}\right)$ as $t \rightarrow t_{m-1}+0$. Therefore,

$$
\int_{t_{m-1}}^{\tau} G_{12} d t \leq C \epsilon^{2 \gamma}
$$

which, together with (8.7), gives

$$
\int_{t_{m-1}}^{\tau}\left\|g_{1}(\cdot, t)\right\|_{\operatorname{pis}(\mathbf{R})} d t \leq C\left(\epsilon^{2 \gamma}+\epsilon^{\gamma(2+\beta)}+\epsilon^{1+\gamma}|\ln \epsilon|\right) .
$$

Next we estimate $\left\|g_{2}\right\|_{\text {pis(R) }}$. Observe that

$$
\begin{aligned}
\left\|g_{2}\right\|_{\operatorname{pis}(\mathbf{R})} & =\int_{2 \epsilon^{\gamma}>\left|x-X_{1}(t)\right| \geq \epsilon^{\gamma}}\left|g_{2}(x, t)\right| d x \leq C \int_{2 \epsilon^{\gamma}>\left|x-X_{1}(t)\right| \geq \epsilon^{\gamma}}\left|V_{1}-H_{1}\right| \epsilon^{-\gamma} d x \\
& =\int_{2>|\xi|>1}\left|V_{1}\left(\xi / \epsilon^{1-\gamma}\right)-H_{1}\left(\xi / \epsilon^{1-\gamma}\right)\right| d \xi \leq C \epsilon^{(1-\gamma) / \beta_{1}} .
\end{aligned}
$$

Therefore,

$$
\int_{t_{m-1}}^{\tau}\left\|g_{2}(\cdot, t)\right\|_{\operatorname{pis}(\mathbf{R})} d t \leq C \epsilon^{(1-\gamma) / \beta_{1}+\gamma}
$$

Similarly, it can be shown that

$$
\int_{t_{m-1}}^{\tau}\left\|g_{3}(\cdot, t)\right\|_{\mathrm{pis}(\mathbf{R})} d t \leq C \epsilon^{(1-\gamma) / \beta_{2}+\gamma} .
$$

We now estimate $\left\|g_{4}\right\|_{\operatorname{pis}(\mathbf{R})}$. Note that

$$
\begin{aligned}
\left\|g_{4}(\cdot, t)\right\|_{\operatorname{pis}(\mathbf{R})} & =\int_{0<\left|x-X_{1}(t)\right|<\epsilon^{\gamma}}+\int_{\left|x-X_{1}(t)\right| \geq \epsilon^{\gamma}}\left|g_{4}(x, t)\right| d x \\
& :=G_{41}+G_{42} .
\end{aligned}
$$

The integrand in $G_{41}$ satisfies the following inequality:

$$
\begin{aligned}
\left|g_{4}\right| \text { in } G_{41}= & \left|a\left(u+\left(V_{1}-H_{1}\right)+m_{2}\left(V_{2}-H_{2}\right)\right)-a\left(V_{1}\right)\right|\left(-V_{1}^{\prime}\right) \epsilon^{-1} \\
\leq & \left|a\left(u+\left(V_{1}-H_{1}\right)+m_{2}\left(V_{2}-H_{2}\right)\right)-a\left(V_{1}+m_{2}\left(V_{2}-H_{2}\right)\right)\right|\left(-V_{1}^{\prime}\right) \epsilon^{-1} \\
& +\left|a\left(V_{1}+m_{2}\left(V_{2}-H_{2}\right)\right)-a\left(V_{1}\right)\right|\left(-V_{1}^{\prime}\right) \epsilon^{-1} \\
:= & \left|g_{41}\right|+\left|g_{42}\right| .
\end{aligned}
$$


The integration of $\left|g_{41}\right|$ is similar to that of (7.13), so it follows from (7.13) that

$$
\int_{\left\{0<\left|x-X_{1}\right| \leq \epsilon^{\gamma}\right\}}\left|g_{41}\right| d x \leq C \epsilon^{\gamma+(1-\gamma) / \beta_{1}} .
$$

We estimate the integral of $g_{42}$ by observing

$$
\begin{aligned}
& \int_{\left\{0<\left|x-X_{1}\right| \leq \epsilon^{\gamma}\right\}}\left|g_{42}\right| d x \\
= & \int_{\left\{\left|x-X_{1}\right| \leq \epsilon^{\gamma}\right\} \cap\left\{\left|x-X_{2}\right| \leq \delta(t) / 2\right\}}\left|g_{42}\right| d x+\int_{\left\{\left|x-X_{1}\right| \leq \epsilon^{\gamma}\right\} \cap\left\{\left|x-X_{2}\right|>\delta(t) / 2\right\}}\left|g_{42}\right| d x \\
:= & G_{411}+G_{412},
\end{aligned}
$$

where $\delta(t):=X_{2}(t)-X_{1}(t)$. Since $\left|g_{42}\right| \leq-C V_{1}^{\prime} \epsilon^{-1}$, we have

$$
\begin{aligned}
G_{411} & \leq C \epsilon^{-1} \int_{\left|x-X_{2}(t)\right| \leq \delta(t) / 2}-V_{1}^{\prime}\left(\left(x-X_{1}(t)\right) / \epsilon\right) d x \\
& \leq C \epsilon^{-1}\left(\frac{2 \epsilon}{\delta(t)}\right)^{1 / \beta_{1}+1} \delta(t) \leq C\left(\frac{\epsilon}{\delta(t)}\right)^{1 / \beta_{1}},
\end{aligned}
$$

which leads to

$$
\begin{aligned}
\int_{t_{m-1}}^{\tau} G_{411} d t & \leq\left. C \epsilon^{1 / \max _{t} \beta_{1}}\left(t-t_{m-1}\right)^{1-1 / \max _{t} \beta_{1}}\right|_{t_{m-1}} ^{\tau} \\
& =C \epsilon^{1 / \max _{t} \beta_{1}}\left(\tau-t_{m-1}\right)^{1-1 / \max _{t} \beta_{1}}
\end{aligned}
$$

where we have applied the inequality (8.4) to the above integration. Since $\tau-t_{m-1} \leq$ $C \epsilon^{\gamma}$ we have

$$
\int_{t_{m-1}}^{\tau} G_{411} d t \leq C \epsilon^{\gamma+(1-\gamma) / \max _{t} \beta_{1}}
$$

On the other hand,

$$
\left|g_{42}\right| \leq C\left|V_{2}-H_{2}\right|\left(-V_{1}^{\prime}\right) \epsilon^{-1}
$$

by the mean value theorem. Substituting this inequality into $G_{412}$ yields

$$
\begin{aligned}
G_{412} & =\int_{\left\{\left|x-X_{1}\right| \leq \epsilon^{\gamma}\right\} \cap\left\{\left|x-X_{2}\right|>\delta(t) / 2\right\}}\left|g_{42}\right| d x \\
& \leq C\left|V_{2}\left(\frac{\delta(t)}{2 \epsilon}\right)-H_{2}\left(\frac{\delta(t)}{2 \epsilon}\right)\right| \int_{-\infty}^{\infty}\left(-V_{1}^{\prime}\left(\left(x-X_{1}\right) / \epsilon\right)\right) \epsilon^{-1} d x \\
& \leq C\left(\frac{2 \epsilon}{\delta(t)}\right)^{1 / \beta_{2}} \int_{-\infty}^{\infty}-V_{1}^{\prime}(\xi) d \xi \leq C\left(\frac{2 \epsilon}{\delta(t)}\right)^{1 / \beta_{2}} .
\end{aligned}
$$

Therefore, on account of $\delta(t) \geq c\left(t-t_{m-1}\right)$ and $\tau-t_{m-1} \leq C \epsilon^{\gamma}$, we obtain

$$
\int_{t_{m-1}}^{\tau} G_{412} d t \leq C \epsilon^{1 / \max _{t} \beta_{2}} \epsilon^{\gamma\left(1-1 / \max _{t} \beta_{2}\right)}=C \epsilon^{\gamma+(1-\gamma) / \max _{t} \beta_{2}} .
$$


This result, together with (8.13) and (8.12), yields

$$
\int_{t_{m-1}}^{\tau} G_{41} d t \leq C \epsilon^{\gamma+(1-\gamma) / \bar{\beta}}
$$

where $\bar{\beta}=\max \left\{\max _{t} \beta_{1}, \max _{t} \beta_{2}\right\}$. In order to estimate $G_{42}$ in (8.11), we first split it into two parts:

$$
\begin{aligned}
G_{42}= & \int_{\left\{\epsilon^{\gamma}<\left|x-X_{1}\right| \leq 2 \epsilon^{\gamma}\right\} \cap\left\{\left|x-X_{2}\right| \leq \delta(t) / 2\right\}} \\
& \quad+\int_{\left\{\epsilon^{\gamma}<\left|x-X_{1}\right| \leq 2 \epsilon^{\gamma}\right\} \cap\left\{\left|x-X_{2}\right|>\delta(t) / 2\right\}}\left|g_{4}\right| d x \\
& :=G_{421}+G_{422} .
\end{aligned}
$$

The integrand $\left|g_{4}\right|$ in $G_{422}$ can be estimated as

$$
\begin{aligned}
\left|g_{4}\right| \text { in } G_{422}= & m_{1}\left|a\left(u+m_{1}\left(V_{1}-H_{1}\right)+m_{2}\left(V_{2}-H_{2}\right)\right)-a\left(V_{1}\right)\right|\left(-V_{1}^{\prime}\right) \epsilon^{-1} \\
\leq & m_{1} \mid a\left(u+m_{1}\left(V_{1}-H_{1}\right)+m_{2}\left(V_{2}-H_{2}\right)\right) \\
& -a\left(H_{1}+m_{1}\left(V_{1}-H_{1}\right)+m_{2}\left(V_{2}-H_{2}\right)\right) \mid\left(-V_{1}^{\prime}\right) \epsilon^{-1} \\
& +m_{1}\left(\left|a\left(H_{1}+m_{1}\left(V_{1}-H_{1}\right)+m_{2}\left(V_{2}-H_{2}\right)\right)-a\left(H_{1}\right)\right|\right. \\
& \left.\quad+\left|a\left(H_{1}\right)-a\left(H_{1}+V_{1}-H_{1}\right)\right|\right)\left(-V_{1}^{\prime}\right) \epsilon^{-1} \\
\leq & C\left(\left|u-H_{1}\right|+\left|V_{1}-H_{1}\right|^{\beta_{1}}+\left|V_{2}-H_{2}\right|^{\beta_{1}}\right)\left(-V_{1}^{\prime}\right) \epsilon^{-1},
\end{aligned}
$$

where we have used the facts that $\left|V_{1}-H_{1}\right| \rightarrow 0$ and $\left|V_{2}-H_{2}\right| \rightarrow 0$ as $\epsilon \rightarrow 0$ for $\left\{\epsilon^{\gamma}<\left|x-X_{1}\right| \leq 2 \epsilon^{\gamma}\right\} \cap\left\{\left|x-X_{2}\right|>\delta(t) / 2\right\}$. Therefore,

$$
\begin{aligned}
G_{422} \leq & C \int_{\left\{\epsilon^{\gamma}<\left|x-X_{1}\right| \leq 2 \epsilon^{\gamma}\right\} \cap\left\{\left|x-X_{2}\right|>\delta(t) / 2\right\}}\left|u-H_{1}\right|\left(-V_{1}^{\prime}\right) \epsilon^{-1} d x \\
& +C \int_{\left\{\epsilon^{\gamma}<\left|x-X_{1}\right| \leq 2 \epsilon^{\gamma}\right\} \cap\left\{\left|x-X_{2}\right|>\delta(t) / 2\right\}}\left|V_{1}-H_{1}\right|^{\beta_{1}}\left(-V_{1}^{\prime}\right) \epsilon^{-1} d x \\
& +C \int_{\left\{\epsilon^{\gamma}<\left|x-X_{1}\right| \leq 2 \epsilon^{\gamma}\right\} \cap\left\{\left|x-X_{2}\right|>\delta(t) / 2\right\}}\left|V_{2}-H_{2}\right|^{\beta_{1}}\left(-V_{1}^{\prime}\right) \epsilon^{-1} d x \\
= & : J_{1}+J_{2}+J_{3} .
\end{aligned}
$$

The estimate for $J_{1}$ is similar to that of $I_{1}$, with an upper bound the same as (7.13), namely,

$$
J_{1} \leq C \epsilon^{\gamma+(1-\gamma) / \beta_{1}}
$$

It follows from (4.9) and (4.10) that

$$
J_{2} \leq C \epsilon^{1-\gamma} \epsilon^{(1-\gamma)\left(1+1 / \beta_{1}\right)} \epsilon^{-1} \epsilon^{\gamma} \leq C \epsilon^{(1-\gamma)\left(1+1 / \beta_{1}\right)} .
$$


Observe that

$$
\begin{aligned}
J_{3}= & C \int_{\left\{\left|x-X_{1}\right| \leq 2 \epsilon^{\gamma}\right\} \cap\left\{\left|x-X_{2}\right|>\delta(t) / 2\right\}}\left|V_{2}-H_{2}\right|^{\beta_{1}}\left(-V_{1}^{\prime}\right) \epsilon^{-1} d x \\
& \leq C\left|V_{2}\left(\frac{\delta(t)}{2 \epsilon}\right)-H_{2}\left(\frac{\delta(t)}{2 \epsilon}\right)\right|^{\beta_{1}} \int_{-\infty}^{\infty}\left(-V_{1}^{\prime}\left(\left(x-X_{1}\right) / \epsilon\right)\right) \epsilon^{-1} d x \\
& \leq C\left(\frac{2 \epsilon}{\delta(t)}\right)^{\beta_{1} / \beta_{2}} \int_{-\infty}^{\infty}-V_{1}^{\prime}(\xi) d \xi \\
& \leq C\left(\frac{2 \epsilon}{\delta(t)}\right)^{\beta_{1} / \beta_{2}} \leq C\left(\frac{2 \epsilon}{\delta(t)}\right)^{1 / \beta_{2}}
\end{aligned}
$$

Therefore, on account of $\delta(t) \geq c\left(t-t_{m-1}\right)$ and $\tau-t_{m-1} \leq C \epsilon^{\gamma}$, we have

$$
\int_{t_{m-1}}^{\tau} J_{3} d t \leq C \epsilon^{1 / \max _{t} \beta_{2}} \epsilon^{\gamma\left(1-1 / \max _{t} \beta_{2}\right)}=C \epsilon^{\gamma+(1-\gamma) / \max _{t} \beta_{2}} .
$$

This, together with (8.17) and (8.18), yields

$$
\int_{t_{m-1}}^{\tau} G_{422} d t \leq C\left(\epsilon^{\gamma+(1-\gamma) / \bar{\beta}}+\epsilon^{(1-\gamma)(1+1 / \bar{\beta})}\right)
$$

where $\bar{\beta}=\max \left\{\max _{t} \beta_{1}, \max _{t} \beta_{2}\right\}$. Since $\left|g_{4}\right| \leq-C V_{1}^{\prime} \epsilon^{-1}$, we have

$$
\begin{aligned}
G_{421} & \leq C \epsilon^{-1} \int_{\left|x-X_{2}(t)\right| \leq \delta(t) / 2}-V_{1}^{\prime}\left(\left(x-X_{1}(t)\right) / \epsilon\right) d x \\
& \leq C \epsilon^{-1}\left(\frac{2 \epsilon}{\delta(t)}\right)^{1 / \beta_{1}+1} \delta(t) \leq C\left(\frac{\epsilon}{\delta(t)}\right)^{1 / \beta_{1}} .
\end{aligned}
$$

Thus

$$
\begin{aligned}
\int_{t_{m-1}}^{\tau} G_{421} d t & \leq\left. C \epsilon^{1 / \max _{t} \beta_{1}}\left(t-t_{m-1}\right)^{1-1 / \max _{t} \beta_{1}}\right|_{t_{m-1}} ^{\tau} \\
& =C \epsilon^{1 / \max _{t} \beta_{1}}\left(\tau-t_{m-1}\right)^{1-1 / \max _{t} \beta_{1}}
\end{aligned}
$$

where we have used the inequality (8.4). Since $\tau-t_{m-1} \leq C \epsilon^{\gamma}$, we have

$$
\int_{t_{m-1}}^{\tau} G_{421} d t \leq C \epsilon^{\gamma+(1-\gamma) / \max _{t} \beta_{1}}
$$

This result, together with (8.20), gives

$$
\int_{t_{m-1}}^{\tau} G_{42} d t \leq C\left(\epsilon^{\gamma+(1-\gamma) / \bar{\beta}}+\epsilon^{(1-\gamma)(1+1 / \bar{\beta})}\right) .
$$

Combining the above result and (8.15) gives

$$
\int_{t_{m-1}}^{\tau}\left\|g_{4}(\cdot, t)\right\|_{\operatorname{pis}(\mathbf{R})} d t \leq C\left(\epsilon^{\gamma+(1-\gamma) / \bar{\beta}}+\epsilon^{(1-\gamma)(1+1 / \bar{\beta})}\right) .
$$

Similarly, it can be shown that

$$
\int_{t_{m-1}}^{\tau}\left\|g_{5}(\cdot, t)\right\|_{\operatorname{pis}(\mathbf{R})} d t \leq C\left(\epsilon^{\gamma+(1-\gamma) / \bar{\beta}}+\epsilon^{(1-\gamma)(1+1 / \bar{\beta})}\right) .
$$


Therefore, we have proved that

$$
\int_{t_{m-1}}^{\tau}\|g(\cdot, t)\|_{\operatorname{pis}(\mathbf{R})} d t \leq C\left(\epsilon^{\gamma+(1-\gamma) / \bar{\beta}}+\epsilon^{(1-\gamma)(1+1 / \bar{\beta})}\right) .
$$

This result and (8.1) yield Lemma 5.4.

9. Numerical experiments. To verify the theoretical results obtained in this work, we shall carry out a computational study in this section. The main purpose is to demonstrate the existence of the fractional rate of convergence. It is generally believed that monotone schemes have the same rate of convergence as that for the viscosity approximation. Therefore, to make the numerical verification available, we consider the (generalized) Lax-Friedrichs scheme

$$
u_{j}^{n+1}=u_{j}^{n}-\frac{\lambda}{2}\left(f\left(u_{j+1}^{n}\right)-f\left(u_{j-1}^{n}\right)\right)+\frac{\mu}{2}\left(u_{j+1}^{n}-2 u_{j}^{n}+u_{j-1}^{n}\right)
$$

to approximate the conservation law (1.1), where $u_{j}^{n}$ is an approximation of $u\left(x_{j}, t_{n}\right)$, and $x_{j}=j \Delta x$, and $t_{n}=n \Delta t$, with $\Delta x$ and $\Delta t$ being the spatial and temporal grid sizes, respectively; $\mu$ is a constant satisfying $0<\mu<1$, and the temporal and spatial grid ratio $\lambda=\Delta t / \Delta x$ satisfies a Courant-Friedrichs-Levy condition,

$$
\lambda \sup _{|u| \leq\left\|u_{0}\right\|_{\infty}}\left|f^{\prime}(u)\right| \leq \mu .
$$

The theoretical properties of the scheme (9.1) were investigated by Liu and Xin [14].

Example 9.1. In the first example, we approximate

$$
\partial_{t} u+\partial_{x} f(u)=0, \quad f(u)=\left(1-u^{2}\right)^{3},
$$

with the initial data $u_{0}(x)=\operatorname{sgn}(x)$, by using the Lax-Friedrichs scheme (9.1).

The entropy solution for the above Riemann problem is $u(x, t)=u_{0}(x)$. It can be verified that $\max _{|u| \leq 1}\left|f^{\prime}(u)\right| \leq 6 / \sqrt{5}$. We then choose $\mu=0.5, T=1$, and $\lambda=\sqrt{5} \mu / 6$. It follows from Theorem 3.1 that the rate of convergence should be $\left(1+\frac{1}{2}\right) / 2=\frac{3}{4}$. It is observed from Table 1 that the numerical rate of convergence agrees very well with the theoretical prediction.

TABLE 1

The $L^{1}$-error and the convergence order for Example 9.1.

\begin{tabular}{||c|c|c|c|c|c||}
\hline Mesh $\Delta x$ & $\frac{1}{8}$ & $\frac{1}{16}$ & $\frac{1}{32}$ & $\frac{1}{64}$ & $\frac{1}{128}$ \\
\hline$L^{1}$-error & $3.22 \times 10^{-1}$ & $2.01 \times 10^{-1}$ & $1.23 \times 10^{-1}$ & $7.44 \times 10^{-2}$ & $4.46 \times 10^{-2}$ \\
\hline Order & & 0.680 & 0.709 & 0.725 & 0.738 \\
\hline
\end{tabular}

Example 9.2. In the second example, we approximate

$$
\partial_{t} u+\partial_{x} f(u)=0, \quad f(u)=(1-u)^{3}(1+u)^{4},
$$

with the initial data $u_{0}(x)=\operatorname{sgn}(x)$, by using the Lax-Friedrichs scheme (9.1).

The entropy solution for the above Riemann problem is again $u(x, t)=u_{0}(x)$. It can be verified that $\max _{|u| \leq 1}\left|f^{\prime}(u)\right| \leq 2$. We then choose $\mu=0.5, T=1$, and $\lambda=$ $\mu / 2$. It follows from Theorem 3.1 that the rate of convergence should be $\left(1+\frac{1}{3}\right) / 2=\frac{2}{3}$. It is observed from Table 2 that the numerical result is again in excellent agreement with the theoretical prediction. 
TABLE 2

The $L^{1}$-error and the convergence order for Example 9.2.

\begin{tabular}{||c|c|c|c|c|c||}
\hline Mesh $\Delta x$ & $\frac{1}{8}$ & $\frac{1}{16}$ & $\frac{1}{32}$ & $\frac{1}{64}$ & $\frac{1}{128}$ \\
\hline$L^{1}$-error & $2.79 \times 10^{-1}$ & $1.89 \times 10^{-1}$ & $1.25 \times 10^{-1}$ & $8.05 \times 10^{-2}$ & $5.11 \times 10^{-2}$ \\
\hline Order & & 0.562 & 0.597 & 0.635 & 0.656 \\
\hline
\end{tabular}

\section{REFERENCES}

[1] D. P. BAllou, Solutions to nonlinear hyperbolic Cauchy problems without convexity conditions, Trans. Amer. Math. Soc., 152 (1970), pp. 441-460.

[2] C. M. Dafermos, Generalized characteristics and the structure of solutions of hyperbolic conservation laws, Indiana Univ. Math. J., 26 (1977), pp. 1097-1119.

[3] C. M. Dafermos, Regularity and large time behavior of solutions of a conservation law without convexity, Proc. Roy. Soc. Edinburgh Sect. A, 99 (1985), pp. 201-239.

[4] J. Goodman, Nonlinear asymptotic stability of viscous shock profiles for conservation laws, Arch. Ration. Mech. Anal., 95 (1986), pp. 325-344.

[5] J. Goodman And Z. XIN, Viscous limits for piecewise smooth solutions to systems of conservation laws, Arch. Ration. Mech. Anal., 121 (1992), pp. 235-265.

[6] P. Howard And K. Zumbrun, Pointwise semigroup methods and stability of viscous shock waves, Indiana Univ. J. Math., 47 (1998), pp. 741-871.

[7] S. N. Krushkov, First-order quasilinear equations with several space variables, Mat. Sb., 123 (1970), pp. 228-255.

[8] N. N. KuzNetsov, Accuracy of some approximate methods for computing the weak solutions of a first-order quasi-linear equation, U.S.S.R. Comput. Math. and Math. Phys., 16 (1976), pp. 105-119.

[9] G. Kreiss and H.-O. Kreiss, Stability of systems of viscous conservation laws, Comm. Pure Appl. Math., 51 (1998), pp. 1397-1424.

[10] P. D. Lax, Hyperbolic systems of conservation laws II, Comm. Pure Appl. Math., 10 (1957), pp. 537-566.

[11] T. P. Liu, Admissible Solutions of Hyperbolic Conservation Laws, Mem. Amer. Math. Soc. 240, AMS, Providence, RI, 1981.

[12] T. P. Liu, Nonlinear Stability of Shock Waves for Viscous Conservation Laws, Mem. Amer. Math. Soc. 328, AMS, Providence, RI, 1985.

[13] T.-P. LiU, Pointwise convergence to shock waves for viscous conservation laws, Comm. Pure Appl. Math., 50 (1997), pp. 1113-1182.

[14] J. LiU AND Z. XIN, $L^{1}$-stability of stationary discrete shocks, Math. Comp., 60 (1993), pp. $233-244$.

[15] A. Matsumura and K. Nishinara, Asymptotic stability of traveling waves for scalar viscous conservation laws with non-convex nonlinearity, Comm. Math. Phys., 165 (1994), pp. 8396.

[16] A. Matsumura and K. Nishinara, On the stability of traveling waves of a one-dimensional model system for compressible viscous gas, Japan J. Appl. Math., 2 (1985), pp. 17-25.

[17] K. Nishinara, Stability of traveling waves with degenerate shock for systems of one-dimensional viscoelastic model, J. Differential Equations, 120 (1995), pp. 304-318.

[18] O. A. Oleinik, Discontinuous solutions of non-linear differential equations, Amer. Math. Soc. Transl., 26 (1963), pp. 95-172.

[19] F. SABAC, The optimal convergence rate of monotone finite difference methods for hyperbolic conservation laws, SIAM J. Numer. Anal., 34 (1997), pp. 2306-2318.

[20] J. A. Smoller, Shock Waves and Reaction-Diffusion Equations, Springer-Verlag, New York, 1984.

[21] A. Szepessy and Z. P. Xin, Nonlinear stability of viscous shock waves, Arch. Ration. Mech. Anal., 122 (1993), pp. 53-103.

[22] E. TAdmor And T. TAng, Pointwise error estimates for scalar conservation laws with piecewise smooth solutions, SIAM J. Numer. Anal., 36 (1999), pp. 1739-1758.

[23] E. TAdmor and T. TAng, Pointwise error estimates for relaxation approximations to conservation laws, SIAM J. Math. Anal., 32 (2000), pp. 870-886.

[24] T. TAng And Z. H. Teng, Viscosity methods for piecewise smooth solutions to scalar conservation laws, Math. Comp., 66 (1997), pp. 495-526. 
[25] T. TAng And Z. H. Teng, The sharpness of Kuznetsov's $O(\sqrt{\Delta x}) L^{1}$-error estimate for monotone difference schemes, Math. Comp., 64 (1995), pp. 581-589.

[26] Z. H. Teng, First-order $L^{1}$-convergence for relaxation approximations to conservation laws, Comm. Pure Appl. Math., 51 (1998), pp. 857-895.

[27] Z. H. Teng And P. Zhang, Optimal $L^{1}$-rate of convergence for the viscosity method and monotone scheme to piecewise constant solutions with shocks, SIAM J. Numer. Anal., 34 (1997), pp. 959-978.

[28] W.-C. WANG, On $L^{1}$ convergence rate of viscous and numerical approximate solutions of genuinely nonlinear scalar conservation laws, SIAM J. Math. Anal., 30 (1998), pp. 38-52.

[29] Z. P. XIN, Theory of viscous conservation laws, in Some Current Topics on Nonlinear Conservation Laws, L. Hsiao and Z. P. Xin, eds., AMS/IP Stud. Adv. Math. 15, AMS, Providence, RI, 2000, pp. 141-194.

[30] Z. P. XIN, Viscous boundary layers and their stability (I), J. Partial Differential Equations, 11 (1998), pp. 97-124.

[31] S.-H. YU, Zero dissipation limit to solutions with shocks for systems of hyperbolic conservation laws, Arch. Ration. Mech. Anal., 146 (1999), pp. 275-370.

[32] K. Zumbrun, Asymptotic Behavior for Systems of Nonconvex Conservation Laws, Ph.D. Dissertation, New York University, New York, 1990. 